\title{
Constant Force Extensional Rheometry of Polymer Solutions
}

\author{
Peter Szabo $^{1 *}$ Gareth H. McKinley ${ }^{2} \quad$ Christian Clasen ${ }^{3}$ \\ ${ }^{1}$ Department of Chemical and Biochemical Engineering, Technical University of Denmark, \\ Søltofts Plads, Building 229, DK-2800 Kongens Lyngby, Denmark; e-mail: ps@kt.dtu.dk \\ 2 Department of Mechanical Engineering, Massachusetts Institute of Technology, \\ Cambridge, Massachusetts 02139, USA; e-mail: gareth@mit.edu \\ ${ }^{3}$ Department of Chemical Engineering, Katholieke Universiteit Leuven,
}

Willem de Croylaan 46, 3001 Heverlee, Belgium; e-mail: christian.clasen@cit.kuleuven.be

September 30, 2011

\begin{abstract}
We revisit the rapid stretching of a liquid filament under the action of a constant imposed tensile force, a problem which was first considered by Matta \& Tytus [JNNFM vol. 35, pp 215-229, 1990]. A liquid bridge formed from a viscous Newtonian fluid or from a dilute polymer solution is first established between two cylindrical disks. The upper disk is held fixed and may be connected to a force transducer while the lower cylinder falls due to gravity. By varying the mass of the falling cylinder and measuring its resulting acceleration, the viscoelastic nature of the elongating fluid filament can be probed. In particular, we show that with this constant force pull (CFP) technique it is possible to readily impose very large material strains and strain rates so that the maximum extensibility of the polymer molecules may be quantified. This unique characteristic of the experiment is analyzed numerically using the FENE-P model and two alternative kinematic descriptions; employing either an axially-uniform filament approximation or a quasi two-dimensional Lagrangian description of the elongating thread. In addition, a second order pertubation theory for the trajectory of the falling mass is developed for simple viscous filaments. Based on these theoretical considerations we develop an expression that enables estimation of the finite extensibility parameter characterizing the polymer solution in terms of quantities that can be extracted directly from simple measurement of the time-dependent filament diameter.
\end{abstract}

\section{Introduction}

The application of a constant force to stretch a viscoelastic liquid sample in order to obtain material properties in extension is very appealing since it simplifies the experimental approach significantly. However, in comparison to shear rheometry in which the

*Author to whom all correspondence should be addressed. 
surface area of a sample to which a force or torque is applied stays constant, in a filament stretching extensional device the sample area (on which a constant force acts) decreases (typically exponentially), resulting in a non-constant stress in the sample. Despite these concerns, Chang \& Lodge [5] have considered the theoretical implications of a constant force pull on a viscoelastic filament in order to determine the 'spinnability' and stability of polymeric liquids in uniaxial extension (due to the rapid approach towards large strains and the finite deformation limit of the polymer network). Matta \& Tytus [28] provided the first experimental results of an extensional rheometry experiment using a constant force pulling on a stretching liquid filament. Their approach utilized a uniform cylindrical fluid sample held between two circular disks with a weight attached to the lower plate which was then released and stretched the fluid filament with a constant gravitational force pull. This groundbreaking work is the progenitor of the filament stretching elongational rheometer (FSR or FiSER) that has been used extensively for evaluating the transient rheometry of mobile liquids. The configuration used by Matta \& Tytus [28] also motivates the experimental setup in the current paper which is depicted in Figure 1. The original apparatus described in [28] was limited in the speed and optical resolution of the monitoring system and did not have the capability to directly measure the force in the extending filament. In the present system we are able to measure the shape of the liquid column every $1 / 2000$ second using high speed video-imaging with a resolution of $\sim 72.3 \mu \mathrm{m} /$ pixel at an image size of $512 \times 1024$ squarepixel.

By using a suitable force balance that incorporated the gravitational body force and monitoring the filament diameter $2 R$, length $L$ of the filament and the actual acceleration of the cylindrical weight, it was possible for Matta \& Tytus [28] to calculate the force (and thus the time varying stress acting in the filament). However, they recognized that it was only possible to obtain 'apparent' transient extensional viscosities as neither the extension rate $\dot{\epsilon}=d \ln L / d t$ nor the axial stress could directly be controlled or kept constant. As in the case of capillary breakup extensional rheometry (CABER) [24, 21, 30, 46, 1, 10], this constant force pull (CFP) technique is not viewed as a true rheometer but more as an indexer for exploring and comparing the extensional behaviour of different liquids. In a capillary thinning device, a thin liquid filament of constant length is stretched beyond its Plateau stability limit and the surface tension (rather than a falling weight) provides the driving force to elongate a liquid filament. In this respect the CFP technique is somewhat more versatile as it allows one to adjust the pulling force by varying the mass of the attached cylinder.

The groundbreaking work of Matta \& Tytus [28] lead to the development of the filament stretching elongational rheometry, as it emphasized the need to develop stretching techniques that allowed one to control and set constant extension rates in order to obtain unambiguous measures of the transient and steady state material properties in uniaxial elongation. First results on filament stretching with controlled uniaxial flow fields were reported by [45] and led to several designs of controlled filament stretching rheometers (FSR) $[2,29,3]$ that have been used extensively for evaluating the transient extensional rheometry of mobile liquids.

For more viscous polymer melts, a number of extensional rheometers were developed by Münstedt, Meissner and coworkers well before the FSR (see [32] for a review). Devices such as the filament stretching apparatus developed by Münstedt et al. [34,35] could rely on a number of assumptions such as negligible effects of capillarity and uniform deformation of molten polymeric samples being elongated, as well as relatively slow stretching dynamics, that allowed one to use realtime measurements of filament length and tensile 
force in order to impose constant rates or stresses. One important observation to note is that the use of fast feedback loops (using the measured forces and filament dimensions in order to determine and control the stress) allowed one to approach a steady state value of the stress much faster than with the controlled strain rates imposed in FSR devices [35]. Recently Sentmanat et al. [41] developed the Sentmanat extensional rheometer (SER) fixture a device, that enables extensional elongation of highly viscous samples with commercially available rotational rheometers. As in the Münstedt device, the SER relies on a controlled deformation applied at the rollers in order to apply an approximately homogeneous strain in the sample $([15])$ and the resulting tensile stress (or resulting torque) is then measured. These devices can also be operated in a constant extensional stress or "tensile creep" mode, by exponentially reducing the tensile force applied to the sample so that the ratio of the force to the time-varying cross-sectional area of the sample remains constant. An interesting observation reported by both Münstedt et al ([35]) and by Sentmanat et al. (2005) ([41]) is that transient extensional rheology experiments operated in tensile creep mode approach steady state more rapidly and at smaller total imposed strain.

As we will show below, this is also true of the Matta \& Tytus falling weight experiment depicted in Fig. 1. This configuration thus provides a simple mechanism for imposing a controlled tensile load to a sample and reaching the steady state elongational viscosity more readily. However, of course, in this experiment it is the externally imposed force that remains constant; the acceleration of the endplate and the total viscoelastic tensile stress exerted by the fluid test sample change as the filament elongates and thins.

In principle, the dripping of a viscoelastic liquid drop from a nozzle can also be used in order to establish a constant tensile force on the thinning filament connecting the pendant drop to the nozzle [18, 19, 42]. Jones et al. [17] used this geometry in conjunction with high speed imaging to explore transient extensional stress growth in the M1 test fluid. An approximate force balance showed that the apparent extensional viscosity could grow by two orders of magnitude as the drop accelerated under gravity. A particularly interesting observation from this early work was that the calculated velocity curves showed the drop to accelerate, to decelerate and then to accelerate again. We observe similar nonmonotonic behavior in the present experiments. Quantifying the kinematics in the falling pendant drop experiment is challenging because the initial filament configuration is not well defined and the mass of the end drop in general does not remain constant during each experiment, but can vary by up to $50 \%$ [17]. Furthermore, variations in the tensile force can only be achieved by increasing the nozzle radius to create larger drops, but this invariably leads also to a larger initial radius of the filament and larger inertial effects. Analysis of the pendant drop experiment by Keiller [19] showed that both viscous and elastic forces contributed significantly to the thinning dynamics and complicated the use of this experiment as a true rheometer. The relatively low mass of a pendant drop (as compared to the weights used by Matta \& Tytus [28] and in the current paper) also results in low imposed deformation rates; for example, the maximum accelerations achieved by Jones et al. were only approximately $\sim 0.5 \mathrm{~g}$. A recent detailed analysis of this problem for the Maxwell/Oldroyd-B model shows that as the tensile stresses grow and retard further acceleration of the pendant droplet and the elongating filament, the dynamics can in fact relax back towards a dominant Newtonian balance [43] which again limits the use of this geometry as an extensional rheometer.

The recent analysis of Smolka et al. [43] highlights a key strength of the CFP technique suggested by Matta \& Tytus: Applying a high enough initial force allows one to impose 
filament deformation rates $d \ln L / d t$ that are sufficiently fast to overcome the relaxation time $\lambda$ and the relaxation processes in the elongating fluid filament. With Weissenberg numbers $W i=\lambda d \ln L / d t \gg 1$ the constant force pull ultimately imposes an almost affine deformation on the microstructural elements in the polymeric sample towards their finite extensibility limit. In contrast to this in capillary breakup (CABER) type experiments the Weissenberg number remains in the elastocapillary balance regime constant at $W i=$ $2 / 3[12,9]$ and the polymeric chains are not deforming affinely with the flow [23, 16]. Although the polymer chains will also in a Caber experiment eventually approach their finite extensibility limit, this requires higher strains than for the affine deformation with the CFP technique. The finite extensibility limit in CABER experiments is therefore typically only at small filament diameters and at the lower resolution limit reached [31, $7,6]$. In principle, affine deformation of polymer chains with the flow field can also be achieved by a filament stretching device. However, the FSR is typically limited in the range of extension rates that can be applied due to the fast feedback loops and accurate force measurements required to achieve constant tensile forces in the filament over distances and velocities similar to the ones covered by the constant force experiments in this paper. The analysis we describe in this paper shows that the combination of a large and constant imposed tensile force on a filament, together with a well-controlled initial rest configuration, enables us to probe directly the finite extensibility limit of dilute polymer solutions in a manner that cannot be achieved with either capillary thinning measurements or filament stretching rheometry.

The problem of a thinning polymer filament being elongated by a constant tensile force is inherently a transient problem in which both the flow kinematics and the bounding domain changes as time progresses. Numerical solution techniques based on a Lagrangian kinematic description should therefore be well suited for the problem considered here. Several formulations exist in literature. For integral constitutive equations of the K-BKZ type, the methods developed by Rasmussen \& Hassager [38], Rasmussen [37] and Marin \& Rasmussen [27] allow for 2D axisymmetric and 3D simulation of K-BKZ type fluids. In addition, for differential constitutive equations, the co-deforming element technique of Harlen et al. [14] and later Morrison \& Rallison [33] has been demonstrated to work for $2 \mathrm{D}$ axisymmetric and $3 \mathrm{D}$ flows. An alternative to these detailed simulation methods is the slender filament approach developed by Renardy [39, 40]. In this technique, the momentum equations are averaged across the filament diameter, thus reducing the effective dimension of the equation set by one. Application of the slender filament technique to studies of filament stretching has been limited by the no-slip condition at solid end-plates which cannot be enforced directly. Here we adopt an idea described by Stokes et al. [47] to help overcome this limitation.

The paper is structured as follows. Section 2 describes the experimental setup of the CFP and the results of experimental measurements of the filament profile and diameter evolution for several different weights and aspect ratios. Section 3 introduces the necessary momentum and force balances in order to numerically simulate the filament profile evolution under the action of a constant force pull. The no-slip boundary condition at the endplates is enforced by an adjustment of the solvent viscosity in the vicinity of the endplates, and polymer stresses are taken into account via the Oldroyd-B and the FENE-P models. The good agreement of the numerical simulations with experimental observations of midfilament diameters and net tensile forces for viscous polymer solutions (as well as for Newtonian threads) justifies the use of the numerical simulations as a benchmark against which simplified analytical solutions are compared. Section 4 introduces a simpli- 
fied force balance for a uniform cylindrical filament that neglects the effects of capillarity and axial curvature. A second order pertubation solution gives an analytical description of the thinning behavior for a Newtonian liquid and can describe the complex evolution in the acceleration of the falling cylinder reported in earlier experiments. This analytical perturbation solution for the Newtonian case, as well as numerical simulations of the simplified uniform filament model that incorporate polymeric stresses, are then compared to the full 1-D simulation in the following section. This uniform filament model provides a nearly quantitative description of the endplate dynamics as a function of Hencky strain when sufficiently large falling masses are used to stretch filaments of dilute polymer solutions. Section 6 uses this simplified model to explore the different thinning dynamics and endplate displacement profiles that are to be expected when critical parameters such as solvent viscosity, endplate mass and finite extensibility limit of the dissolved polymer are systematically varied. The final section of the paper focuses on the possibility of using a transition in the thinning dynamics of the filament in order to provide an approximate probe of the finite extensibility of the polymer chains that initially deform affinely with the fluid under a constant force pull.

\section{Experimental setup and measurements}

A schematic of the experimental setup is shown in Fig. 2. Experiments were conducted using two parallel aluminum plates of radius $R_{0}=5.12 \times 10^{-3} \mathrm{~m}$ between which the fluid sample was loaded. The upper, stationary plate was connected to the force transducer (405A, Aurora Scientific Inc, Ontario, Canada) of a filament stretching rheometer (FSR) with a sensitivity of $2 \times 10^{-5} \mathrm{~g}$ and a response time of $1 \times 10^{-3} \mathrm{~s}$. Weights with nominal masses of $1 \mathrm{~g}, 2 \mathrm{~g}, 5 \mathrm{~g}, 10 \mathrm{~g}$, and $20 \mathrm{~g}$ were glued to the lower aluminum plate. The total mass of the lower plate plus the attached cylindrical weight and glue was determined prior to each experimental run. The combined assembly of lower plate and cylinder is initially supported by the lower stage of the FSR. Prior to an experimental run, the adjustable lower stage of the FSR was used to adjust the initial plate separation $L_{0}$ in order to set a desired aspect ratio $\Lambda=L_{0} / R_{0}$ (experiments were run at three different nominal aspect ratios of $\Lambda=0.2,0.3$ and 0.5 ). Samples were then loaded between the plates using a syringe to accurately fill the gap. The position of the lower stage was then adjusted in order to achieve an exact cylindrical shape with sample radius $R_{0}$. The exact aspect ratios $\Lambda$ obtained after the position correction of the lower plate are reported for each experimental run. The lower stage of the FSR, supporting the lower plate assembly, was then accelerated downwards with an acceleration of $1.2 \mathrm{~g}$ in order to instantly release the lower plate assembly. This release mechanism assured a clean separation of the lower plate/cylindrical weight assembly from the lower retaining stage of the FSR without any associated momentum transfer. The travel distance of the falling cylinder and therefore the final stretch length of the liquid filament was limited by the final displacement of the lower stage of the FSR to $L_{\max }=1.3 \mathrm{~m}$.

The evolution of the liquid filament stretched between the two circular plates by the falling cylinder, as well as the position of the cylinder was monitored with a high speed camera (Phantom 5, Vision Research Inc.) at a frame rate of 2000 fps and with a spatial resolution of $72.3 \mu \mathrm{m} /$ pixel and field of view of $512 \times 1024 \mathrm{pixel}^{2}$. The maximum length of the liquid filament that was possible to monitor at this magnification (from the top plate to the lower boundary of picture) was $6.81 \times 10^{-2} \mathrm{~m}$. The filament radius as a function of 
radial position, $R(z)$, was determined for each individual frame using a self-written image processing algorithm (LabView Vision, National Instruments) [8]. The determination of the filament diameter was limited by the uneven diffusive backlighting distribution at this large field of view and resulted in an error for the outline of the filament of \pm 0.5 pixel. All reported filament diameters are therefore limited to $D>4.5 \times 10^{-4} \mathrm{~m}$. For filament lengths smaller then $0.14 \mathrm{~m}$ (twice the directly observable filament length) the absolute minimum mid-filament radius $R_{\text {mid }}$ could be obtained directly for each frame from the $R(z)$ data. These values were limited to diameters in the range of $1.4 \times 10^{-3} \mathrm{~m}$ for the largest initial aspect ratios, down to approximately $0.9 \times 10^{-3} \mathrm{~m}$ depending on the initial aspect ratio of the liquid bridge. Below this limit (that is also indicated in the following experimental Figs. 3-5) the reported minimum diameters do not represent the actual value measured at the mid-position between the two endplates; however, because the viscoelastic filament profile is almost cylindrical (see the example shown in Fig. 1), the observable filament radius at the lower edge of the image is a very close approximation to the actual minimum.

The total force acting on the top plate was determined with a sampling frequency of $112 \mathrm{~Hz}$, the reported data in the figures represent floating averages over time intervals of $0.1 \mathrm{~s}$. The initial reference time for the force data is directly correlated with the movement of the lower retaining plate, and the initial motion could be synchronized with the video data of the diameter evolution to within $0.01 \mathrm{~s}$.

\subsection{Fluid properties}

The fluid used in the present study consisted of a poly(styrene) standard with a molecular weight of $M_{w}=2.84 \times 10^{6} \mathrm{~g} / \mathrm{mol}$ and a polydispersity of $P=1.13$ (obtained from Polymer Standard Services, Ontario, NY) dissolved in styrene oligomers (Picolastic A5 Resin) at a concentration of $c_{w}=0.025 \mathrm{wt} \%$ (so-called Boger fluid). The zero-shear viscosity of this fluid (as well as the linear viscoselastic moduli) was determined with a rotational rheometer (AR2000, TA Instruments) to be $\eta_{0}=92$ Pas.

The modulus $G$ of a dilute solution of monopisperse linear homopolymers can be calculated from the number concentration $n$ of the polymer coils as $G=n k_{B} T$, which is easily obtained from the weight concentration $c_{w}$ and the molecular weight of the polymer $M$ via $n=c_{w} N_{A} / M$. For the present fluid we obtain $G=2.2 \times 10^{-1} \mathrm{~Pa}$.

Assuming a discrete Zimm relaxation spectrum as appropriate for dilute polymer solutions [36], the longest relaxation time $\lambda$ is obtained from fitting experimental linear viscoelastic moduli $G^{\prime}(\omega)$ and $G^{\prime \prime}(\omega)$ with the expressions

$$
\begin{aligned}
G^{\prime} & =\frac{\eta_{s} \lambda_{s} \omega^{2}}{1+\left(\lambda_{s} \omega\right)^{2}}+G \sum_{i=1}^{N_{\text {modes }}} \frac{(\lambda \omega)^{2}}{i^{4+2 \tilde{\sigma}}+(\lambda \omega)^{2}} \\
G^{\prime \prime} & =\frac{\eta_{s} \omega}{1+\left(\lambda_{s} \omega\right)^{2}}+G \sum_{i=1}^{N_{\text {modes }}} \frac{(\lambda \omega) i^{2+\tilde{\sigma}}}{i^{4+2 \tilde{\sigma}}+(\lambda \omega)^{2}}
\end{aligned}
$$

where $\omega$ is the angular frequency and $\eta_{s}$ and $\lambda_{s}$ are the viscosity and relaxation time of the oligomeric solvent respectively [26]. Values of $\tilde{\sigma}=-0.445$ as a measure of the hydrodynamic interaction and $N_{\text {modes }}=8$ for the number of relevant modes for a Boger fluid were obtained for a similar system in [10]. The longest relaxation time of the fluid used in this study was found to be $\lambda=24.8 \mathrm{~s}$. 
The effective viscosity $\eta_{s}$ of the solvent in the solution is then determined from eq. (2) at low frequencies, $\eta_{0}=\eta_{s}+1.86 G \lambda$, and is calculated in the present case to be $\eta_{s}=86.5$ Pas.

The finite extensibility $b$ for a dilute suspension of monodisperse linear homopolymer chains can be computed from first principles as

$$
b=3\left[\frac{\sin ^{2}\left(\frac{\theta}{2}\right) M_{w}}{C_{\infty}\left(M_{u} / j\right)}\right]^{2(1-\nu)} .
$$

Here $j$ is the number of bonds along the chain per monomeric unit, $\theta$ is the bond angle, $C_{\infty}$ is the characteristic ratio, $\nu$ is the excluded volume exponent and $M_{w}$ and $M_{u}$ are the weight average molecular weight of the polymer and the molecular weight of the monomeric unit in the chain. With $j=2$ and $\theta=109.4^{\circ}$ for an aliphatic chain, $C_{\infty}=$ 9.6 and $\nu=0.52$ for polystyrene in the athermal solvent of styrene oligomers, and with a monomeric molecular weight of $M_{u}=104 \mathrm{~g} / \mathrm{mol}$ the finite extensibility parameter is calculated to $b=8176$.

The surface tension $\sigma$ of the fluid is $0.035 \mathrm{~N} / \mathrm{m}$, determined with a Krüss K10ST Tensiometer (Hamburg, Germany).

A polydimethylsiloxane (PDMS) oil was used as a Newtonian reference fluid having a constant viscosity of $\eta_{s}=61.4 \mathrm{Pas}$ and a surface tension of $\sigma=0.02 \mathrm{~N} / \mathrm{m}$.

The appropriate values of non-dimensional fluid parameters used in this study can then

be calculated from the physical parameters: the capillary number is $C a=\left(3 \eta_{s} R_{0} / \sigma\right) \sqrt{g / R_{0}}$ (with $C a=2064$ for the PDMS oil and $C a=1551$ for the polymer solution); the Deborah

number of the polymer solution is $D e=\lambda \sqrt{g / R_{0}}=1085$ and the dimensionless polymer concentration is $c=G \lambda / \eta_{s}=0.063$.

\section{$2.2 \quad$ Stretching experiments}

In the following section we present measured data for the evolution of the midfilament diameter $2 R_{\text {mid }}(t)$ from several stretching experiments with the polymer solution described above. The experiments have different loads and initial aspect ratios. Figure 3 shows the evolution of the minimum filament diameter versus time for four different loads (weights of $1.8 \mathrm{~g}, 2.8 \mathrm{~g}, 5.8 \mathrm{~g}$ and $20.8 \mathrm{~g}$ ). The initial aspect ratios are rather small; of the order $\Lambda=0.2$. We observe a very characteristic behaviour in which the diameter of the filament thins increasingly rapidly due to the constant force imposed by the falling cylindrical weight, but then at a critical point in time there is a change in slope and the thinning process deviates from the expected Newtonian behaviour (see Figure 9 for comparison).

As illustrated in Figure 4 this behaviour persists at larger aspect ratios $(\Lambda \simeq 0.3)$. We note, however, that this transition region (where the slope $\mathrm{d} R / \mathrm{d} t$ changes rapidly) becomes increasingly diffuse with increasing weight and larger aspect ratios (see Figure 5). At the largest aspect ratios and weights shown in Figure 5 this transition point is difficult to identify at all.

A more intuitive way to represent this data is the apparent acceleration of the falling cylinder that one can obtain from the filament diameter evolution. Assuming a cylindrical configuration the apparent length of the filament is $L_{a p p}=L_{0} \pi R_{0}^{2} / \pi R_{m i d}^{2}$. This apparent length has been differentiated twice after a Savitzky-Golay smoothing. The weighted averages of this second derivative $d^{2} L_{a p p} / d t^{2}$, normalized by the gravitational accelaration 
$g=9.81 \mathrm{~m} / \mathrm{s}^{2}$, are given in Figures 6 and 7 as a function of the apparent Hencky strain $\ln \left(L_{\text {app }} / L_{0}\right)$. If there was no fluid resistance then the cylinder should fall freely and this reduced accelaration should approach a constant value of 1 . The apparent initial overshoot of the acceleration at Hencky strains $\sim 2$ is due to the no-slip boundary condition at the end plates and the resulting non-cylindrical, hourglass shape of the filament in the beginning. Due to volume conversation the mid-filament diameter $2 R_{m i d}$ is initially decreasing faster than for a purely cylindrical configuration of the filament, leading to an acceleration of the apparent length $L_{a p p}=L_{0} \pi R_{0}^{2} / \pi R_{\text {mid }}^{2}$ that exceeds $g$. This effect is increasing with mass (Fig. 6) and with increasing aspect ratio (Figure 7). Despite the differences in the initial accelaration, it is obvious that independent of the pulling weight or the aspect ratio we observe for all experiments an onset of a strong deceleration at similar Hencky strains of $\ln \left(L_{a p p} / L_{0}\right) \sim 2.4$ with a minimum at $\ln \left(L_{\text {app }} / L_{0}\right) \sim 2.9$.

\section{Simulation of stretch in non-uniform filaments un- der a constant force pull}

\subsection{Momentum balance}

We consider a slender axisymmetric filament described by the radius, $R(z, t)$, as a function of the axial position $z$ as depicted in Figure 2. In the following we shall neglect fluid inertia and the effect of gravity on the fluid filament itself, i.e. we assume that the Reynolds number, $R e \ll 1$ and that the ratio mass(fluid)/mass(solid) $\ll 1$. Gravity will, however, appear in the force balance for the falling solid cylindrical weight. In the limit of creeping flow, the radial and axial components of the momentum equation become (see [4]):

$$
\begin{aligned}
0 & =\frac{\partial \pi_{r r}}{\partial r}+\frac{\left(\tau_{r r}-\tau_{\theta \theta}\right)}{r}+\frac{\partial \tau_{z r}}{\partial z} \\
0 & =\frac{1}{r} \frac{\partial}{\partial r}\left(r \tau_{r z}\right)+\frac{\partial \pi_{z z}}{\partial z}
\end{aligned}
$$

where $\boldsymbol{\pi}=p_{a} \boldsymbol{\delta}+\boldsymbol{\tau}$ is the total stress composed of a pressure and a deviatoric stress $\boldsymbol{\tau}$.

At the filament surface we apply a stress boundary condition of the form [22]

$$
\boldsymbol{n} \cdot \boldsymbol{\pi}=\left(p_{a}+2 H \sigma\right) \boldsymbol{n}
$$

which at a free surface, $R(z)$, reduces to the following shear and normal stress boundary conditions (a derivation of the expressions $\boldsymbol{n}$ and $2 H$ may be found in [48]):

$$
\begin{aligned}
\pi_{r r}-R^{\prime} \tau_{z r} & =p_{a}+2 H \sigma \\
\tau_{r z}-R^{\prime} \pi_{z z} & =-\left(p_{a}+2 H \sigma\right) R^{\prime}
\end{aligned}
$$

Here $p_{a}$ is the external pressure, $\sigma$ is the (constant) surface tension and $2 H$ is the mean curvature given by:

$$
2 H=\frac{1 / R}{\left(1+\left(R^{\prime}\right)^{2}\right)^{1 / 2}}-\frac{R^{\prime \prime}}{\left(1+\left(R^{\prime}\right)^{2}\right)^{3 / 2}}=\frac{1}{R R^{\prime}} \frac{\mathrm{d}}{\mathrm{d} z}\left(\frac{R}{\sqrt{1+\left(R^{\prime}\right)^{2}}}\right)
$$

In the expressions above, $R$ denotes the radial position $R(z)$, while $R^{\prime}$ and $R^{\prime \prime}$ refer respectively to the axial derivatives $d R / d z$ and $d^{2} R / d z^{2}$ at the same position. 
We average the axial momentum equation (5) across a surface perpendicular to the $z$-axis and obtain:

$$
\begin{aligned}
0 & =-\int_{0}^{R(z)} \frac{1}{r} \frac{\partial}{\partial r}\left(r \tau_{r z}\right) r \mathrm{~d} r-\int_{0}^{R(z)} \frac{\partial \pi_{z z}}{\partial z} r \mathrm{~d} r \\
& =-R\left[\tau_{r z}\right]_{R}-\frac{\partial}{\partial z} \int_{0}^{R} \pi_{z z} r \mathrm{~d} r+\left[\pi_{z z}\right]_{R} R^{\prime} R
\end{aligned}
$$

From the free surface condition in equation (8) we take the axial contribution,

$$
\left[\tau_{r z}\right]_{R}-R^{\prime}\left[\pi_{z z}\right]_{R}=-\left(p_{a}+2 H \sigma\right) R^{\prime}
$$

and obtain

$$
0=R R^{\prime}\left(p_{a}+2 H \sigma\right)-\frac{\partial}{\partial z} \int_{0}^{R} \pi_{z z} r \mathrm{~d} r
$$

We rewrite $\pi_{z z}$ in the form $\pi_{r r}+\left(\tau_{z z}-\tau_{r r}\right)$, use integration by parts and insert the equations (4), (7) and (9) to obtain the following equation.

$$
\begin{array}{r}
0=\frac{\partial}{\partial z}\left[\frac{\sigma R}{2 \sqrt{1+\left(R^{\prime}\right)^{2}}}\left(1+\frac{R R^{\prime \prime}}{1+\left(R^{\prime}\right)^{2}}\right)-\int_{0}^{R}\left(\tau_{z z}-\tau_{r r}\right) r \mathrm{~d} r\right. \\
\left.-\frac{1}{2} \int_{0}^{R}\left(\tau_{r r}-\tau_{\theta \theta}\right) r \mathrm{~d} r-\frac{1}{2} \frac{\partial}{\partial z} \int_{0}^{R}\left(r \tau_{z r}\right) r \mathrm{~d} r\right]
\end{array}
$$

The details of this derivation are similar to those described in [48]. The above equation may be integrated once to yield

$$
\begin{aligned}
F(t)= & \frac{\sigma R}{2 \sqrt{1+\left(R^{\prime}\right)^{2}}}\left(1+\frac{R R^{\prime \prime}}{1+\left(R^{\prime}\right)^{2}}\right)-\frac{R^{2}}{2}<\tau_{z z}-\tau_{r r}> \\
& -\frac{R^{2}}{4}<\tau_{r r}-\tau_{\theta \theta}>-\frac{\partial}{\partial z}\left(\frac{R^{2}}{4}<r \tau_{z r}>\right)
\end{aligned}
$$

where $<. .>$ represent averages over cross-section defined by the integrals above. Provided the material is initially in equilibrium and has not been pre-sheared, the average value $<\tau_{r r}-\tau_{\theta \theta}>$ will vanish in elongational flow. The term $\left\langle r \tau_{z r}>\right.$ also vanishes in regions of strong elongational flow. It becomes, however, the dominant stress term close to the end-plates because of the no-slip boundary condition (see discussion in [48] and [50]). In the following, therefore, we shall assume that the terms $\left\langle\tau_{r r}-\tau_{\theta \theta}\right\rangle$ and $\left\langle r \tau_{z r}\right\rangle$ can be neglected in the majority of the elongating fluid filament. As described below, a special procedure will be used to model the mixed 2D flow close to the end-plates.

The above equation (15) is now reduced to

$$
2 F(t)=\frac{\sigma R}{\sqrt{1+\left(R^{\prime}\right)^{2}}}\left(1+\frac{R R^{\prime \prime}}{1+\left(R^{\prime}\right)^{2}}\right)-R^{2}<\tau_{z z}-\tau_{r r}>
$$

which is equivalent to equation (8) in [39]. The above equation (16) is, however, more accurate for the description of curvature in non-uniform filaments. The use of higher order descriptions of surface curvature to accurately capture the free surface dynamics of Newtonian filaments and jets is discussed by Eggers [11]. 
In addition to the momentum balance (16) an equation is needed to ensure mass conservation. We follow the Lagrangian approach of Renardy [39] and introduce the average stretch, $s$, of an axial element defined by

$$
s\left(z_{0}, t\right)=\frac{\partial z}{\partial z_{0}}=\left(\frac{R\left(z_{0}, t_{0}, t_{0}\right)}{R\left(z_{0}, t_{0}, t\right)}\right)^{2}
$$

where $z_{0}$ is the initial axial reference position of a material element and $t_{0}$ is a reference time (i.e. the time when the cylinder is released). An equivalent compact expression is

$$
R=\frac{R_{0}}{\sqrt{s}}
$$

for each material element $s\left(z_{0}, t\right)$. Introducing the stretch into equation (16) we obtain

$$
2 F(t)=\frac{\sigma R_{0}}{\sqrt{s}}\left[\frac{1}{\sqrt{1+\left(R^{\prime}\right)^{2}}}\left(1+\frac{R R^{\prime \prime}}{1+\left(R^{\prime}\right)^{2}}\right)\right]-\frac{R_{0}^{2}}{s}<\tau_{z z}-\tau_{r r}>
$$

where the Eulerian derivatives of $R(z)$ are replaced by Lagrangian measures for $s\left(z_{0}\right)$ as follows

$$
R^{\prime}=\frac{\partial}{\partial z} R\left(z_{0}, t_{0}, t\right)=-\frac{R_{0}}{2} s^{-5 / 2} \frac{\partial s}{\partial z_{0}}
$$

and

$$
R R^{\prime \prime}=R \frac{\partial^{2}}{\partial z^{2}} R\left(z_{0}, t_{0}, t\right)=5\left(R^{\prime}\right)^{2}-\frac{R_{0}^{2}}{2} s^{-4} \frac{\partial^{2} s}{\partial z_{0}^{2}}
$$

We now replace the average stress difference $\left\langle\tau_{z z}-\tau_{r r}>\right.$ by a sum of contributions from a Newtonian solvent and a polymeric solute undergoing elongational flow. Hence

$$
<\tau_{z z}-\tau_{r r}>=-3 \eta_{s} \frac{\partial v_{z}}{\partial z}+\left(\tau_{p, z z}-\tau_{p, r r}\right)=-\frac{3 \eta_{s}}{s} \frac{\partial s}{\partial t}+\left(\tau_{p, z z}-\tau_{p, r r}\right)
$$

which we insert into equation (19) and rearrange to obtain the following form,

$$
3 \eta_{s} R_{0}^{2} \frac{\partial s}{\partial t}=2 F(t) s^{2}+R_{0}^{2} s\left(\tau_{p, z z}-\tau_{p, r r}\right)-\sigma R_{0} s^{3 / 2} \mathcal{C}(s)
$$

where $\mathcal{C}(s)$ represents the curvature term in square brackets in (19).

The time dependent total axial force $F(t)$ acting between the endplates is determined by integration as follows: For a filament having initial length $L_{0}$, we note that (see [39])

$$
\int_{0}^{L_{0}} \frac{\partial s\left(z_{0}, t_{0}, t\right)}{\partial t} \mathrm{~d} z_{0}=\frac{\partial}{\partial t} \int_{0}^{L_{0}} s\left(z_{0}, t_{0}, t\right) \mathrm{d} z_{0}=\frac{\mathrm{d} L}{\mathrm{~d} t}
$$

where $L(t)$ is the actual filament length at time $t$. Integrating equation (23) over each material element along the filament we obtain

$$
2 F(t)=\frac{\int_{0}^{L_{0}}\left\{\sigma R_{0} s^{3 / 2} \mathcal{C}(s)-R_{0}^{2} s\left(\tau_{p, z z}-\tau_{p, r r}\right)\right\} \mathrm{d} z_{0}+3 \eta_{s} R_{0}^{2} \frac{\mathrm{d} L}{\mathrm{~d} t}}{\int_{0}^{L_{0}} s^{2} \mathrm{~d} z_{0}}
$$

A similar expression was given in [26]. The evolution of the filament is determined from the combined solution of equation (23) for each material element $s\left(z_{0}, t\right)$ and (25) for the total force over all material elements, in conjunction with the force balance for a constant force pull and a viscoelastic constitutive equation with appropriate boundary and initial conditions. The discretization is uniform in the Lagrangian co-ordinate $z_{0}$ with typically 800-1600 elements along the filament. 


\subsection{Force balance}

The global force balance necessary to solve for the evolution of the filament is obtained from the experimentally-observable acceleration of the lower endplate/mass assembly shown in Figure 1. The acceleration, described by the actual filament length $\mathrm{d}^{2} L / \mathrm{d} t^{2}$, is determined by the gravitational acceleration of the endplate assembly mass $m$ and the resisting force $F(t)$ (eq. (25)) in the filament. Newton's second law for the falling mass can then be written in the form

$$
m \frac{\mathrm{d}^{2} L}{\mathrm{~d} t^{2}}=m g-F(t)
$$

where we neglect any contribution from aerodynamic drag on the falling mass [17]. The differential equation is augmented by the initial conditions $L(0)=L_{0}$ and $\dot{L}(0)=0$. Furthermore, with $F(0)=0$ it follows from eq. (26) that $\ddot{L}(0)=g$. In the present implementation we use a simple forward Euler integration method to integrate eq. (26) and evaluate the falling plate velocity $\mathrm{d} L / \mathrm{d} t$ needed in eq. (24)-(25). The maximum time step is determined by the stability of the scheme.

\subsection{The no-slip boundary condition at the axial end plates}

In previous studies where a similar slender filament methodology was applied [39, 40, 51, 26 the no-slip condition was not strictly enforced at the end plates. The slender filament equations comprise a 'far field' solution which must be patched to a 'near field' solution close to the end plates. Without this near field solution, an initial cylindrical filament with zero polymer stresses and $\dot{L}=0$ remains stationary. It is therefore difficult to unambiguously connect the evolving stress in the filament at long times with the initial rest configuration shown in the first image of Figure 1. Here we adopt an alternative technique proposed by Stokes et al. [47]. They studied a cylindrical sample of Newtonian fluid draining from a solid surface due to gravity. At the solid-liquid surface a no-slip condition must be imposed. This was achieved by modifying the effective viscosity locally so that it becomes increasingly large as we approach the solid surface. This correction is not arbitrary but derived such that close to a solid surface a local 2D lubrication flow (or reverse squeeze film flow as described in [44]) is obtained while far from the solid surface no correction is applied and the extensional viscosity approaches the expected value $3 \eta_{s}$. Stokes et al. [47] proposed an empirical expression for the local variation in the viscosity which is equivalent to

$$
3 \eta_{s}^{*}=3 \eta_{s}\left[1+\frac{1}{8}\left(\frac{R_{0}}{\Delta z}\right)^{2}\right]
$$

with $\Delta z$ being the distance to the solid surface. In the present study a geometry having two rigid end-plates is considered. We therefore apply a slightly modified expression for the modified viscosity,

$$
3 \eta_{s}^{*}=3 \eta_{s}\left[1+\frac{1}{a}\left\{\left(\frac{R_{0}}{\Delta z_{1}}\right)^{2}+\left(\frac{R_{0}}{\Delta z_{2}}\right)^{2}\right\}\right]
$$

where $\Delta z_{1}$ and $\Delta z_{2}$ refer to the actual distances from a specific material point (with label $\left.z_{0}\right)$ to the two end-plates. The value of the parameter $a$ will vary with the initial aspect ratio $\Lambda=L_{0} / R_{0}$ and needs to be determined. A comparison of the surface shape produced 
by the numerical simulation using the condition (28) with $a=55.8$ to an experimentally observed shape is shown in Fig. 8 (this value of $a$ corresponds to the empirical choice $32 / \Lambda$ which is used in all simulations). It is clear from Fig. 8 that the surface shape obtained from simulation is very similar to the experimental observations.

An expression for the surface positions in the lubrication limit, $R(z, t)$, can be derived analytically (see [48]):

$$
R(z, t)=R_{0}\left[\left(\frac{L}{L_{0}}\right)+\left(\frac{2 z}{L}-1\right)^{2}\left(1-\frac{L}{L_{0}}\right)\right]^{-3 / 4}
$$

Here, $z=0$ corresponds to the upper fixed plate in Fig. 2 while the point $z=L(t)$ is located at the lower falling plate. A comparison of the surface shape produced by equation (29) to the experimental data is also given in Fig. 8 for $\Lambda=L_{0} / R_{0}=0.57$. As expected the lubrication approximation does not capture the deformation of the free surface near the endplate as accurately as the numerical simulation .

\subsection{Constant force thinning of Newtonian fluids}

Solving equations $(23),(25)$ and (26) with the polymer stress contribution $\left(\tau_{p, z z}-\tau_{p, r r}\right)$ set to zero and with the modified viscosity of (28), we can calculate the evolution of a thinning Newtonian liquid filament under a constant force pull. A comparison of the calculated midfilament diameter evolution as well as the force $F(t)$ to actual experimental data is given in Figs. 9a and $9 \mathrm{~b}$. The midfilament radius $R\left(z_{0}=0.5 L_{0}, t\right)$ initially thins slowly as the lower plate starts to fall under gravity. As the distance between the plates increases, the viscous lubrication forces become progressively smaller and the falling plate accelerates. After 0.8 seconds the midpoint radius has decreased by a factor of 30; the Hencky strain experienced by a material element at the midplane is thus $\epsilon_{\text {mid }}=2 \ln \left(R_{0} / R_{\text {mid }}\right) \approx 6.8$. The force exerted on the upper endplate is given by eq. 25 (with $\tau_{p, z z}-\tau_{p, r r}=0$ ). The force recorded by the stationary transducer in the upper plate rises very rapidly to a plateau value given by $F_{\sigma}=m g$. As the filament thins and the falling mass accelerates, the transmitted force rapidly drops to zero. The plate is then

in 'free fall' with $\ddot{L}=g$. The very small magnitude of the transmitted force makes the measurements more susceptible to noise than direct observations of the filament radius.

\subsection{Polymer solutions}

When the liquid filament connecting the falling mass to the stationary upper plate is a polymer solution we need to incorporate the polymeric stresses $\left(\tau_{p, z z}-\tau_{p, r r}\right)$ in $(23)$ and (25) that were neglected in the previous section. For dilute polymer solutions we consider the elastic stresses predicted by the Oldroyd-B and FENE-P models (see [4] for reference). We may express the components of the polymer stress in terms of the non-dimensional structure tensor $\mathbf{A}$ :

$$
\boldsymbol{\tau}_{p}=-G[h(\operatorname{tr} \mathbf{A}) \mathbf{A}-\mathbf{I}]
$$

Here $G=n k T$ is the elastic modulus of the solution. The function $h(\operatorname{tr} \mathbf{A})$ is defined by

$$
h(\operatorname{tr} \mathbf{A})=\left\{\begin{array}{cl}
1 & \text { Oldroyd-B } \\
(1-\operatorname{tr} \mathbf{A} / b)^{-1} & , \text { FENE-P }
\end{array}\right.
$$


where $\operatorname{tr} \mathbf{A}=A_{i i}$ is the trace of the structure tensor, and $b$ is the finite extensibility parameter characterizing the maximum extension of the polymer chain $(\lim (\operatorname{tr} \mathbf{A}) \rightarrow b)$. The components of the structure tensor are governed by a set of evolution equations:

$$
\frac{\partial \mathbf{A}}{\partial t}+\mathbf{v} \cdot \nabla \mathbf{A}-\nabla \mathbf{v}^{T} \cdot \mathbf{A}-\mathbf{A} \cdot \nabla \mathbf{v}=-\frac{1}{\lambda}[h(\operatorname{tr} \mathbf{A}) \mathbf{A}-\mathbf{I}]
$$

where $\lambda$ is the characteristic time constant of the material. As described above we use a Lagrangian description of the stretching flow with the Lagrangian stretch $s\left(z_{0}, t\right)$ accounting for the deformation. Thus, in a geometry with radial symmetry and zero initial stress we may reduce the equations in (32) to the following two equations for each element $(i)$ :

$$
\begin{aligned}
& \frac{\mathrm{d} A_{r r}^{(i)}}{\mathrm{d} t}=\frac{1}{\lambda}\left[1-A_{r r}^{(i)} h\left(\operatorname{tr} \mathbf{A}^{(i)}\right)\right]-\dot{\epsilon}^{(i)}(t) A_{r r}^{(i)} \\
& \frac{\mathrm{d} A_{z z}^{(i)}}{\mathrm{d} t}=\frac{1}{\lambda}\left[1-A_{z z}^{(i)} h\left(\operatorname{tr} \mathbf{A}^{(i)}\right)\right]+2 \dot{\epsilon}^{(i)}(t) A_{z z}^{(i)}
\end{aligned}
$$

where $\dot{\epsilon}^{(i)}(t)$ is the extensional strain-rate in the $i^{t h}$ material element

$$
\dot{\epsilon}^{(i)}=-\frac{2}{R}\left(\frac{\partial R}{\partial t}\right)_{z_{0}}=\frac{1}{s}\left(\frac{\partial s}{\partial t}\right)_{z_{0}}
$$

and the trace of the structure tensor is $\operatorname{tr} \mathbf{A}^{(i)}=A_{z z}^{(i)}+2 A_{r r}^{(i)}$. It should be noted that equations (33) and (34) must be solved for each Lagrangian element $z_{0}$ in a discretisation.

We can now solve equations $(23),(25),(26)$ with (28) and the polymer stress contribution $\left(\tau_{p, z z}-\tau_{p, r r}\right)$ taken into account via (30) and the evolution equations (33)-(35). A comparison of these numerical simulations to experimental data for the FENE-P model is shown in Figures 10 and 11 for two different initial masses. In each case the falling cylindrical weight initially accelerates slowly due to the large viscous lubrication forces in the small gap. As the mass accelerates, the midpoint radius decreases more rapidly as was also observed for the Newtonian fluid. However, at a critical point, corresponding to a radius reduction of $R_{\text {mid }} / R_{0} \sim 0.2$, the force measured by the transducer suddenly increases, the falling mass decelerates and the rate of thinning in the midfilament diameter radius is dramatically reduced. At longer times the force decays again and the acceleration of the falling mass picks up once more. These complex dynamics are consistent with those reported by Matta \& Tytus [28] and Jones et al. [17]. As we discuss in the following section, the sudden growth in the elastic stress and the deceleration of the falling plate may be considered as akin to that of a "viscous bungee jumper" falling under gravity. In order to understand the key features of this response in a falling cylinder experiment we need to consider the equations of motion (23)-(28), (30) and (33)-(35) in more detail.

The near quantitative comparison of the numerical simulations with experimentally

observed midfilament diameters and forces in the filament of Newtonian fluid coupled with the good agreement for the polymer solutions suggest that we can use the numerical simulations as a benchmark against which the simplified analytical solutions of the following sections can be compared.

\section{A simple force balance for a uniform filament: the viscous bungee jumper}

In addition to solving the full set of $1 \mathrm{D}$ radially averaged equations represented by equations $(25,33-34)$ for the filament shape $s\left(z_{0}, t\right)$, and stresses $\mathbf{A}\left(z_{0}, t\right)$ we introduce in the 
following section a simpler model problem that still captures the key physical features of the falling plate rheometer but allows for analytical solutions of the problem. Again we consider a uniform cylindrical liquid bridge of length $L_{0}$ positioned between a fixed plate and a weight having mass $m$. At some initial time $t_{0}$ the weight is released and starts falling due to gravity. However, for simplicity we do not enforce the no-slip boundary at the endplates and assume the filament remains cylindrical through the thinning process. Figure 1 suggests this should be a good approximation and the plate falls in the same way as a "bungee jumper" attached to a stationary support. However, in contrast to a true bungee jumper (connected by a purely elastic rope) who is in free fall until the cord snaps taut, the viscous bungee jumper's fall is slowed by the (rate and strain-dependent) tensile stress in the elongating fluid thread.

Furthermore, we neglect the effects of surface tension in the cylindrical filament and also neglect the effects of fluid inertia (but not, of course, the inertia of the falling mass). The total force acting on the falling cylinder may then be equated to the gravitational body force minus the resistance in the viscoelastic filament (or 'bungee cord') due to viscous and/or elastic stresses (see [48, 49]). We obtain the following equation for the length of the filament, $L(t)$.

$$
m \frac{\mathrm{d}^{2} L}{\mathrm{~d} t^{2}}=m g+<\tau_{z z}-\tau_{r r}>\pi R^{2}
$$

where the last term has a positive sign because of our choice of sign convention that tensile stresses are negative (Bird et al. [4]). We may express the stresses in the polymer solution as a sum of contributions from a Newtonian solvent and the polymeric solute. Hence

$$
m \frac{\mathrm{d}^{2} L}{\mathrm{~d} t^{2}}=m g+\left[-3 \eta_{s} \dot{\epsilon}+\left(\tau_{p, z z}-\tau_{p, r r}\right)\right] \pi R^{2}
$$

In the cylindrical fluid filament the volume is conserved both locally and globally. Thus $R_{0}^{2} L_{0}=R^{2} L$ and $\dot{\epsilon}=\dot{L} / L=-2 \dot{R} / R$ for each material element.

\subsection{Effect of capillarity}

Before further developing the uniform filament model we note that ignoring capillarity and the effects of surface tension can lead to considerable deviations at short times from the actual thinning dynamics that are observed in experiments and in the full numerical simulations. In order to quantify the effect of capillarity on the thinning dynamics we must first non-dimensionalize the governing equations. The natural timescale for these falling cylinder experiments is $t_{g}=\sqrt{R_{0} / g}$ corresponding to a strain rate $\dot{\epsilon}_{g} \sim \sqrt{g / R_{0}}$. The characteristic viscous force is then $F_{v} \sim 3 \eta_{s} \dot{\epsilon}_{g} R_{0}^{2}$ and substituting these scalings in equations (23) and (25) results in the following expressions

$$
\frac{\partial s}{\partial \tau}=2 F^{*}(\tau) s^{2}+\frac{c}{3 D e} s \frac{\left(\tau_{p, z z}-\tau_{p, r r}\right)}{G}-\frac{1}{C a} s^{3 / 2} \mathcal{C}(s)
$$

and

$$
2 F^{*}(\tau)=\frac{\int_{0}^{\Lambda}\left\{\frac{1}{C a} s^{3 / 2} \mathcal{C}(s)+\frac{c}{3 D e} s \frac{\left(\tau_{p, z z}-\tau_{p, r r}\right)}{G}\right\} \mathrm{d} \zeta_{0}+\frac{\mathrm{d} \zeta}{\mathrm{d} \tau}}{\int_{0}^{\Lambda} s^{2} \mathrm{~d} \zeta_{0}}
$$


with the non-dimensional force $F^{*}(\tau)=F(t) / F_{v}=F(t) /\left(R_{0}^{2} 3 \eta_{s} \sqrt{g / R_{0}}\right)$, dimensionless filament length

$$
\zeta=L(t) / R_{0}
$$

the non-dimensional time

$$
\tau=t / \sqrt{R_{0} / g}
$$

and the polymer contribution to the viscosity

$$
c=G \lambda / \eta_{s}
$$

The dimensionless Lagrangian position is defined by $\zeta_{0}=z_{0} / R_{0}$. The ratio of the polymer relaxation time $\lambda$ to the gravitational time scale results in a Deborah number

$$
D e=\lambda \sqrt{g / R_{0}}
$$

and the ratio of viscous stresses to the surface pressure $\sigma / R_{0}$ gives the capillary number

$$
C a=\frac{3 \eta_{s} R_{0}}{\sigma} \sqrt{\frac{g}{R_{0}}} .
$$

With the physical parameters of the polymer solution used in the experiments in Figure 10 we obtain a value of $C a=1551$ (and $C a=2064$ for the Newtonian PDMS). Although these values are large, solving (38) and (39) for the Newtonian case and different values of $\mathrm{Ca}$, we can see in Figure 12 from the numerical simulations for the surface shape at a particular time $(\tau=39.5)$ that varying the capillary number can have a pronounced effect; the surface shapes and total axial displacement at capillary numbers $C a=100$ and $C a=1000$ are very different. Physically, this is because at short times the filament is not long and slender, and neither is the initial fluid velocity of $O\left(\dot{\epsilon}_{g} R_{0}\right)$ as implied by eq. (44). For $1000 \leq C a \leq 10000$ (the range corresponding to the experimental capillary numbers) the difference is much smaller, but still not negligible. This observation indicates that neglecting capillary effects for the uniform filament model will result in a small deviation from the numerical results in the temporal evolution of the filament, particularly at early times.

\subsection{The viscous Newtonian fluid bungee jumper}

Using the above definitions for the non-dimensional variables in the force balance (37) of the uniform filament model leads (for a Newtonian fluid) to the simple nonlinear ordinary differential equation

$$
\frac{\mathrm{d}^{2} \zeta}{\mathrm{d} \tau^{2}}+\frac{V}{\zeta^{2}} \frac{\mathrm{d} \zeta}{\mathrm{d} \tau}=1
$$

which describes the time-evolution in the bungee jumper's position. Here we have defined another non-dimensional number $V$ as:

$$
V=\frac{3 \pi \eta_{s} L_{0}}{m} \sqrt{\frac{R_{0}}{g}}
$$


The parameter $V$ can be interpreted as a ratio of the viscous force in the filament $3 \eta_{s}\left(\pi R_{0}^{2}\right) \dot{\epsilon}_{g}$ and the gravitational force $m g$ multiplied by the aspect ratio $\left(L_{0} / R_{0}\right)$. In our experiments we can span values of $V$ in the range from 0.7 to about 20 by changing the mass $m$ of the falling cylinder.

We may integrate equation (45) once to obtain,

$$
\frac{\mathrm{d} \zeta}{\mathrm{d} \tau}-\frac{V}{\zeta}=\tau+C_{1}
$$

The constant of integration, $C_{1}$, is determined from the appropriate initial conditions. We require no flow initially with the specified aspect ratio, $\Lambda=L_{0} / R_{0}$, i.e.

$$
\zeta(0)=\Lambda \quad \text { and } \quad \frac{\mathrm{d} \zeta}{\mathrm{d} \tau}(0)=0
$$

Equation (47) then becomes,

$$
\frac{\mathrm{d} \zeta}{\mathrm{d} \tau}-\frac{V}{\zeta}=\tau-\frac{V}{\Lambda}
$$

This deceptively simple first order nonlinear ODE has no simple analytical solution that we are aware of, but can be integrated numerically for any initial values of $V$ and $\Lambda$. In addition, we derive a pertubation solution in the parameter $V$. Thus, we expand $\zeta(\tau)$ as

$$
\zeta=\zeta_{0}+V \zeta_{1}+V^{2} \zeta_{2}+\ldots
$$

where the zeroth order term $(V=0)$ corresponds to the free fall of a weight with no viscous retarding force (or a bungee jumper with no rope!) and therefore gives rise to a displacement profile:

$$
\zeta_{0}=\frac{1}{2} \tau^{2}+\Lambda
$$

i.e. in absence of any viscous fluid stress, the cylinder accelerates quadratically in time as expected. The higher order corrections to eq. (50) may be derived with increasing levels of algebraic manipulations. We find that the first and second order correction terms are necessary to describe with sufficient accuracy the displacement of the falling cylinder for typical experimental values of $V$. They become,

$$
\zeta_{1}=-\frac{\tau}{\Lambda}+\sqrt{\frac{2}{\Lambda}} f
$$

and

$$
\zeta_{2}=\frac{5}{4 \Lambda^{2}}-\frac{1}{\Lambda\left(\Lambda+\tau^{2} / 2\right)}-\frac{1}{2 \Lambda^{2}}\left[f^{2}+f \sin 2 f+\frac{1}{2} \cos 2 f\right]
$$

where

$$
f=\operatorname{Arctan}\left(\frac{\tau}{\sqrt{2 \Lambda}}\right)
$$

Differentiating equation (50) repeatedly with respect to non-dimensional time $\tau$ we may derive useful expressions for the velocity and the acceleration of the falling cylinder. We obtain the following expression for the first and second time derivatives, i.e. the velocity

$$
\frac{\mathrm{d} \zeta}{\mathrm{d} \tau}=\tau-\frac{V}{\Lambda} \frac{(\tau / \sqrt{2 \Lambda})^{2}}{\left[1+(\tau / \sqrt{2 \Lambda})^{2}\right]}+\frac{V^{2}}{\Lambda} \frac{\sqrt{2 / \Lambda}}{\left[1+(\tau / \sqrt{2 \Lambda})^{2}\right]^{2}}\left(\frac{\tau}{\sqrt{2 \Lambda}}-\operatorname{Arctan}\left(\frac{\tau}{\sqrt{2 \Lambda}}\right)\right)
$$


and the acceleration

$$
\frac{\mathrm{d}^{2} \zeta}{\mathrm{d} \tau^{2}}=1-\frac{V}{\Lambda^{2}} \frac{\tau}{\left[1+(\tau / \sqrt{2 \Lambda})^{2}\right]^{2}}\left\{1+\frac{V}{\Lambda} \frac{3(\tau / \sqrt{2 \Lambda})-4 \operatorname{Arctan}(\tau / \sqrt{2 \Lambda})}{\sqrt{2 \Lambda}\left[1+(\tau / \sqrt{2 \Lambda})^{2}\right]}\right\}
$$

From the expression in Eq. (56) we see clearly that the initial acceleration is $\ddot{\zeta}(0)=1$ but also that $\ddot{\zeta}(\tau) \rightarrow 1$ for $\tau \rightarrow \infty$, i.e. as the cross-sectional area of the filament decreases, the ability of the viscous stress to retard the acceleration of the cylinder decreases correspondingly.

The approximate physics captured by the pertubation solution can be appreciated from the plots in Fig. 13 and Fig. 14. We observe that for values of $V \leq 0.1$ the 1st order pertubation solution (i.e. the first two terms of eq. (50)) is very accurate (Fig. 13). For values between $0.1 \leq V \leq 1.0$ the extent of viscous deceleration at intermediate times $(\tau \sim O(1))$ is larger and the 2nd order pertubation solution is required for an accurate representation of the solution (see Fig. 14). The pertubation solution in eqs. (51)-(53) can be applied for $V$-values slightly larger than order unity but higher order approximations which are not small corrections to the lower order terms become increasingly important.

\subsection{Oldroyd-B and FENE behaviour}

We now extend the analysis of the uniform filament model to include polymeric stresses. With the definition (30) for the polymer stress contribution, equation (37) becomes:

$$
\frac{\mathrm{d}^{2} \zeta}{\mathrm{d} \tau^{2}}=1-\frac{V}{\zeta}\left[\frac{1}{\zeta} \frac{\mathrm{d} \zeta}{\mathrm{d} \tau}+h(\operatorname{tr} \mathbf{A})\left(A_{z z}-A_{r r}\right) \frac{c}{3 D e}\right]
$$

again using the non-dimensional parameters corresponding to a polymer viscosity, $c=$

$G \lambda / \eta_{s}$, and a Deborah number, $D e=\lambda \sqrt{g / R_{0}}$. For the cylindrical filament with equilibrium initial conditions, equations (33) and (34) become:

$$
\begin{aligned}
& \frac{\mathrm{d} A_{r r}}{\mathrm{~d} \tau}=-\frac{1}{\zeta} \frac{\mathrm{d} \zeta}{\mathrm{d} \tau} A_{r r}+\frac{1}{D e}\left[1-h(\operatorname{tr} \mathbf{A}) A_{r r}\right] \\
& \frac{\mathrm{d} A_{z z}}{\mathrm{~d} \tau}=+\frac{2}{\zeta} \frac{\mathrm{d} \zeta}{\mathrm{d} \tau} A_{z z}+\frac{1}{D e}\left[1-h(\operatorname{tr} \mathbf{A}) A_{z z}\right]
\end{aligned}
$$

The equations $(57,58,59)$ are solved subject to the initial conditions $\zeta(0)=\Lambda, d \zeta / d \tau(0)=$ 0 and $A_{r r}(0)=A_{z z}(0)=b /(b+3)(\sim 1$ for the Oldroyd-B model in which $b \rightarrow \infty)$. In the following section we compare the predictions of these simplified equations with full numerical calculations.

\section{Comparison of the uniform filament model and the no-slip model}

We have shown in Figs. 13 and 14 that for a Newtonian fluid with relevant parameter values the simple uniform filament model can be reproduced accurately by a second order pertubation solution. In the following we compare numerical solutions of the uniform filament model for polymer solutions with the full 1-dimensional Lagrangian model described above in section 3. As the initial stage of the stretching experiment is dominated by the lubrication flow and the no-slip boundary condition illustrated in Fig. 8 we do 
not expect the evolution in the dimensionless radius function $R(t) / R_{0}$ of the uniform filament model to be predicted well for short times (when the Hencky strain is below order unity). In Fig. 15a this is seen clearly for a FENE-P model fluid. We observe, as expected, that the one-dimensional Lagrangian filament thins faster at the middle due to the no-slip condition at the plates. If we additionally consider the acceleration of the bottom plate as depicted in Fig. 15b we gain some further insight. The plate motion can be divided into three distinct regions: an early viscous behaviour (as described by the pertubation solution above), followed by a sudden transition and deceleration, and finally an asymptotic approach to gravitational acceleration. This behaviour indicates that there is a rapid transition from viscous to elastic behaviour. The plate initially accelerates rapidly, resulting in rapid stretching of the polymer; however, the elastic stress does not contribute to the dominant force balance. As the polymer chains become highly stretched the elastic stresses grow rapidly to resist further stretching of the filament. At this point the 'bungee cord' tautens and the falling cylinder experiences a strong negative (i.e. upwards) acceleration. It is worth noting that the uniform filament model predicts the qualitative behaviour of the falling plate; the details of the dynamics are, however, delayed due to the neglect of capillarity and the no-slip boundary, as demonstrated in Fig. 12 .

An alternative choice to plotting results as a function of dimensionless time $\tau=$ $t \sqrt{g / R_{0}}$ is to use the accumulated Hencky strain, $\ln (\zeta / \Lambda)$, of the material element at the centerplane $\left(z_{0}=0.5\right)$ as an independent variable. Then, as shown in Fig. 16a, we observe immediately that the uniform filament model can predict very accurately the sequence of transitions in the falling plate motion as a function of apparent strain. To further qualify this conclusion we show in Fig. 16b the polymer stretch measure trA versus Hencky strain. The predicted evolution in the stretch from the uniform filament model is everywhere very close to the Lagrangian model (except at the point of maximum stretch and at very high strains). The other observation that becomes immediately apparent from Fig. $16 \mathrm{~b}$ is that the molecular deformation accumulated by the material element at the midplane becomes very large and $\operatorname{tr} \mathbf{A}$ approaches the maximum extensibility (i.e. $\operatorname{tr} \mathbf{A} \rightarrow b$ ) at a very moderate Hencky strain $\epsilon \sim 3.5$. We now proceed to examine this rapid stretching limit in more detail.

\section{Affine deformation of the polymer under a con- stant force pull}

We have shown above that the uniform filament model is a useful approximation for understanding the rapid stretching flow in a falling cylinder device when the accumulated strain rather than the elapsed time is used as the appropriate variable to parameterize the filament evolution. This observation is consistent with the high Deborah numbers $D e \gg 1$ in the flow (for example for the experiments in Figs. 3-5 the Deborah number is calculated

to be $\left.D e=\lambda \sqrt{g / R_{0}}=1085\right)$. In the following section we explore the behaviour of this simplified model for several combinations of the non-dimensional parameters $V, \Lambda, b, c$ and $D e$. We consider first the acceleration $\ddot{\zeta}$ in a uniform FENE-P filament. In Fig. 17a we show for $1 \leq V \leq 6.6$ the behaviour of the acceleration. (In our experiments we span values of $V$ in the range from 0.76 to 22 by changing the mass $m$ of the falling cylinder). Smaller values of $V$ produce qualitatively similar curves as $V=1$. For short times $\tau \leq 5$ 
we observe a behaviour similar to a Newtonian fluid (compare to Fig. 14). Then a sudden decrease is observed in the acceleration - which becomes increasingly negative before it eventually approaches again the gravitational value corresponding to $\ddot{\zeta}=1$ at long times. To gain a deeper understanding of the behaviour we show in Fig. 17b the acceleration versus the apparent Hencky strain (for $\ln (\zeta / \Lambda) \geq 3$ ) and again observe that the sudden deceleration occurs at almost identical values of the accumulated strain. A seemingly strange effect occurs for $V=6.6$ in the approach to gravitational acceleration. The acceleration becomes a non-monotonous function of the strain which indicates a bouncing or recoil behaviour due to the interplay of fluid elasticity and the inertia of the falling mass, an effect similar to that observed in a true bungee jumper!

If we consider also the evolution in polymer stretch, $\operatorname{tr} \mathbf{A}$, as shown in Fig. 18, we observe that at the critical strain $\epsilon^{*}$ corresponding to strong deceleration we have, at the same time, almost fully stretched polymer chains i.e. $\operatorname{tr} \mathbf{A} \rightarrow b$. Furthermore, the evolution of $\operatorname{tr} \mathbf{A}$ in Fig. 18 before reaching $\epsilon^{*}$ is independent of the applied weight $V$. Because of the very large value of the Deborah number in these constant force experiments there is an almost affine deformation of the polymer chains in the deforming filament. The Weissenberg numbers $W i=\lambda \dot{\epsilon}$ during the inital acceleration phase is $W i \gg 1$ (and the maximum Weissenberg number $W i_{\max }$ is directly related to $D e$, see details in Appendix II) so that there is little possibility of relaxation before reaching $\epsilon^{*}$. As the filament is stretched, the individual polymers are elongated, and elastic stresses build up in the filament. As the chains reach full elongation and snap taut the falling motion is rapidly arrested and the falling cylinder stops or even starts to bounce depending on the choice of parameters. A similar observation was made by Keiller [19] who studied a falling drop of an Oldroyd-B fluid.

In Fig. 19 we see this behaviour clearly. The filament radius is here shown versus non-dimensional time for a Newtonian fluid as well as for FENE-P and Oldroyd-B fluids. The curve for the Newtonian fluid may be taken as a reference as it is indistinguishable (on these scales) from the result of a freely falling body, i.e. $\zeta=\Lambda+0.5 \tau^{2}$ and therefore $R / R_{0}=\left[1+\tau^{2} /(2 \Lambda)\right]^{-1 / 2}$. We observe that the FENE-P fluids (which have a finite extensibility) reach a critical point at which the filament thinning is brought to an almost complete stop. Beyond this point, stress relaxation commences and the filament starts to extend once more due to the constant imposed weight. Careful examination of Fig. 18 shows that in this region $(\epsilon>4)$ the chains do in fact relax slowly, even though the plate is still falling; we examine this asymptotic regime in more detail below. The Oldroyd-B fluid has no finite extensibility. Hence material elements can become highly extended before the viscoelastic stresses have grown to a level where they drive a recoil (bouncing) in the plate position. A similar effect is observed for FENE-P fluids having a very large $b$-value.

\section{Experimental determination of the finite extensi- bility from falling cylinder experiments}

Another interesting observation that may be made in Fig. 18 is that the polymer stretch $\operatorname{tr} \mathbf{A}$ at larger strains becomes independent of $V$. The asymptotic behaviour of tr $\mathbf{A}$ may in fact be predicted by a theory developed from simple assumptions. Because $D e \gg 1$ (and $W i \gg 1$ ) and the polymer chains become almost fully stretched, the extensional viscosity reaches the steady state value expected at large strain rates. This allows for the use of a 
pertubation solution with the dimensionless parameter $V$ replaced by the value $V_{\infty}$ based on the elongational viscosity in steady uniaxial elongation. We apply the pertubation solution to first order in $V$ from Eq. (56) and obtain

$$
\frac{\mathrm{d}^{2} \zeta}{\mathrm{d} \tau^{2}} \sim 1-\frac{4 V_{\infty} \tau}{\left(2 \Lambda+\tau^{2}\right)^{2}}
$$

where $V_{\infty}=V(1+2 c b / 3)$ is based on the steady extensional viscosity at large Deborah numbers (see details in Appendix I). Combining (60) with the non-dimensional stress balance of eq. (57) and an assumption of $\zeta(\tau)=\Lambda+0.5 \tau^{2}$ (i.e. the free fall expression), we obtain

$$
h(\operatorname{tr} \mathbf{A})\left(A_{z z}-A_{r r}\right) \frac{c}{3 D e}=\frac{4 c b}{3\left(2 \Lambda+\tau^{2}\right)}
$$

which decays monotonically at long times. We further assume that $\tau^{2} \gg 2 \Lambda$ and $A_{z z} \gg$ $A_{r r}$ and $A_{z z} \approx \operatorname{tr} \mathbf{A}$. Inserting then the expression $h(\operatorname{tr} \mathbf{A})=(1-\operatorname{tr} \mathbf{A} / b)^{-1}$ from eq. (31) we obtain after a few manipulations an expression for the evolution in the scalar quantity $\operatorname{tr} \mathbf{A}(\tau)$ :

$$
\operatorname{tr} \mathbf{A}(\tau)=\frac{4 b D e}{4 D e+\tau}
$$

This equation may be expressed in terms of the strain by inverting the expression for the free fall trajectory $\zeta(\tau)$ and substituting in eq. (62). This asymptotic solution (which assumes Newtonian-like filament thinning dynamics for a dilute solution of fully stretched chains in order to predict the evolution of the chain extension towards full stretch) is then compared to simulations in Fig. 18. We observe that the simulated stretch curves follow the asymptotic result up to an apparent Hencky strain of $\epsilon=8$. Beyond this point, the stretch has relaxed so much that some of the assumptions leading to the asymptotic result in eqs. (61)-(62) start to fail.

To further understand the asymptotic behaviour of the unraveling polymer chains in the falling cylinder device, we compare in Fig. 20 the evolution of $\operatorname{tr} \mathbf{A}$ from the numerical simulations of the uniform filament model to the limiting case of polymer chains that deform affinely with a fluid element without relaxation. Here we take $V=1$ because this value results in minimal overshoot or elastic recoil when $A_{z z} \rightarrow b$ (see Fig. 18). Evidently the simulations show that constant force extension does not allow (for high enough $V$ and $D e$ values) the polymer to relax and the molecular deformation follows the fluid deformation until the polymer stretch has almost reached the maximum stretch, $\operatorname{tr} \mathbf{A} \rightarrow b$. Beyond this point the polymer stretch stays almost constant at $b$ for larger axial strains.

This observation of affine deformation motivates a simple analysis from which we may estimate the strain at which the maximum stretch is achieved. This information can then be used to extract the FENE stretch parameter $b$ from measured position data, i.e. it can be read directly from the point of onset of the shoulder in the radius versus time plot in Fig. 19 (or Figs. 3-5).

The analysis is as follows. In equation (59) we assume that $h(\operatorname{tr} \mathbf{A}) \approx 1$ and that $A_{z z} \gg 1$. This simplification leads to the following equation (63).

$$
\frac{1}{A_{z z}} \frac{\mathrm{d} A_{z z}}{\mathrm{~d} \tau}=\frac{2}{\zeta} \frac{\mathrm{d} \zeta}{\mathrm{d} \tau}-\frac{1}{D e}
$$


The large values of the Deborah number $D e=\lambda \sqrt{g / R_{0}} \gg 1$ in the experiments and corresponding simulations suggest that we can simplify this equation even further to obtain the time-independent expression in equation (64).

$$
\frac{\mathrm{d} \ln A_{z z}}{\mathrm{~d} \ln \zeta}=2
$$

Integrating this equation with the appropriate boundary conditions yields the following expression for an affine polymer deformation with the flow:

$$
\ln A_{z z}(\tau)=2 \ln (\zeta / \Lambda)
$$

Combining this expression with $\operatorname{tr} \mathbf{A}=A_{z z}+2 A_{r r}$ and the expectation that $A_{r r} \approx O(1)$ in a strong uniaxial elongational flow gives

$$
\operatorname{tr} \mathbf{A} \approx 2+(\zeta / \Lambda)^{2}
$$

This expression is shown by the solid line in Fig. 20. The maximum polymer stretch corresponds to $A_{z z} \rightarrow b$ and equating this value with eq. (66) leads to the following expression for the critical Hencky strain that is imposed by the falling endplate,

$$
\ln \left(\zeta_{b} / \Lambda\right)=\frac{1}{2} \ln b
$$

This expression can also be related to a characteristic cylinder radius in the uniform filament model which is given by the very simple expression

$$
R_{b} / R_{0}=b^{-1 / 4}
$$

where $R_{b}$ is the radius for which a shoulder begins to form, as apparent in Fig. 10 and Fig. 19 or in the experimental data of Figure 3 - 5. This simple expression is independent of the mass of the falling cylinder, or the initial aspect ratio of the test sample. Of course the time required to achieve this strain does depend on the other dimensionless parameters in the problem and must be determined from simulation or asymptotic analysis.

Inverting the expression in equation (68) shows that the FENE extensibility parameter $b$ is directly related to the filament radius at the 'pinch point' corresponding to the appearence of a shoulder, i.e.

$$
b_{\text {exp }} \approx\left(R_{0} / R_{b}\right)^{4}
$$

The large numerical value of the exponent in this expression suggests that minimizing noise in measurements of the critical radius $R_{b}$ is critical. From Fig. 19 we note, however, that experimentally we only have to resolve values for $R_{b}$ of the order $0.1 R_{0}(\approx 100 \mu \mathrm{m})$ which is relatively easy. As an example we consider the experimental diameter versus time data presented in Figs. 3-5. From these data sets it is apparent that the transitional shoulder indeed is independent of the applied weight or aspect ratio and, for this fluid, occurs always between values of $R_{b} \simeq 1.0 \pm 0.05 \mathrm{~mm}$. This value translates into an estimated $b$-value between 565 and 844 . This value is smaller than the theoretical value obtained from purely molecular parameters in section 2.1. The discrepancy is not surprising given that it is now well known that a FENE-P model substantially overpredicts the true tensile stress in strong elongational flows [20, 13]. Furthermore, as laid out by Hsieh and Larson [16] the effective hydrodynamic drag on a high molecular weight polymer will not 
be constant as assumed in the discussion above, but will depend on the degree of stretching of the polymer coil. The translational drag coefficient for the polymer molecules will increase with increasing deformation. The effective b-value at the transition can therefore be smaller as the one based on molecular theory, as this 'conformation-dependent' drag is likely to significantly retard the chain unraveling and this will further retard the acceleration of the falling mass. However, this stresses even more the versatility of the simple constant force pull in comparison to other rheometric techniques for a more direct estimate for the appropriate value of $b_{\text {exp }}$ characterizing a polymer solution [25]. 


\section{Conclusions}

In this paper we have revisited the falling cylinder experiment first described by Matta \& Tytus [28] which has been used by a number of investigators to probe the transient extensional viscosity of mobile polymer solutions. Unlike the dripping of a pendant drop, the ability to systematically vary the weight of the cylindrical mass enables one to control the deformation conditions in the fluid thread. Unfortunately, because the flow in the elongating filament is inhomogeneous in space and time, it is difficult to use such a device to measure the true uniaxial elongational viscosity function as one does with a filament stretching rheometer. However, our analysis and experiments show that this simple experiment can be used in a different way to make a direct estimate of the effective maximum extensibility of the polymeric chains dissolved in the solution. Specifically, because the appropriate Deborah number $D e=\lambda / \sqrt{R_{0} / g}$ characterizing this constantforce extensional flow is so high, the Weissenberg number $W i \gg 1$ and the deformation of the material is initially affine in character. The slender nature of the geometry makes analysis of this time-dependent free-surface problem particularly well-suited to a onedimensional Lagrangian formulation. In addition we have shown that a simplified 'uniform cylinder approximation' enables the problem to be reduced to a set of coupled ordinary differential equations that can accurately capture the kinematics in the elongating fluid thread. The resulting dynamics of the falling mass are analogous to that of a bungee jumper connected to a rigid inertial frame by a viscoelastic fluid rope.

For a purely viscous fluid, a perturbation analysis enables us to calculate the initial non-monotonic variation in the acceleration $\ddot{L}$ that was documented in early experiments $[28,17]$. When released, the mass attached to the cylindrical endplate is initially in free fall for a short time; however the viscous resisting force increases with the fall velocity and this decelerates the endplate. At later times this viscous force decreases as the crosssectional area of the elongating thread is reduced and the falling cylinder thus begins to accelerate again. When polymer chains are present, the affine deformation in the initial accelerating phase results in rapid and large molecular elongations. Eventually the polymer chains snap taut and the polymeric contribution to the stress grows very rapidly, resulting in a second sudden deceleration in the endplate trajectory, as well as coupled inertio-elastic oscillations of the cylindrical mass. In the final stages of the process the bungee jumper accelerates once more, but not sufficiently fast to prevent the polymer chains from slowly relaxing. Our experiments and numerical simulations show that the critical 'break point' at which the polymer chains first snap taut (denoted by a strain $\epsilon_{b}$ or equivalently a thread radius $R_{b}$ ) can be detected either by the deceleration of the falling endplate or by a marked change in the rate of necking in the long thin bungee rope.

The simplified uniform cylinder analysis indicates that the Hencky strain at which this critical point occurs only depends, to a first approximation, on the finite extensibility of the chains as given by eq. (68). Exploratory calculations with the one dimensional Lagrangian formulation show that the uniform cylinder approximation is most closely realized for initial sample aspect ratios $\Lambda \sim O(1)$ and the inertio-elastic oscillations are minimized for dimensionless masses $V \simeq 1$. The resulting value of the finite extensibility parameter are within an order of magnitude of the ideal value calculated from first principles using the manufacturers data for the polymer molecular weight. In the affine stretching limit that is consistent with this constant tensile force device, the total Hencky strain required to achieve maximum molecular stretch only depends on the $1 / 4^{\text {th }}$ power of the extensibility parameter. The resulting strains are thus surprisingly moderate, corre- 
sponding to radius reductions of only factors of 10-30. They are therefore relatively easy to observe, provided a high speed imaging system is available and a reliable mechanism is developed for cleanly releasing the lower plate and attached cylindrical weight (in our experience this is not trivial to achieve). The falling cylinder device may thus serve a useful role for quickly establishing constitutive parameters relevant to low viscosity polymer solutions at large deformation rates and large strains.

\section{Acknowledgements}

The financial support from Otto Mønsteds Fond and the Danish Technical Research Council (grant 26-03-0191) to allow Peter Szabo to visit MIT during the summer of 2004 is gratefully acknowledged. Christian Clasen acknowledges financial support of this work from the ERC starting grant GA n 203043 - NANOFIB. Additional support for research in the Non-Newtonian Fluids lab at MIT is provided by gifts from Schlumberger Doll Research and Procter \& Gamble. 


\section{Appendix I: Steady state elongation of a FENE-P fluid}

Assuming steady state in Eqs. (33) and (34) we obtain after rearrangement the following algebraic equations:

$$
\begin{aligned}
& 1=A_{r r}(h(\operatorname{tr} \mathbf{A})+\dot{\epsilon} \lambda) \\
& 1=A_{z z}(h(\operatorname{tr} \mathbf{A})-2 \dot{\epsilon} \lambda)
\end{aligned}
$$

where $\operatorname{tr} \mathbf{A}=A_{z z}+2 A_{r r}$ at steady state. Combining these expressions we obtain implicit expression for $\operatorname{tr} \mathbf{A}$ as a function of $\lambda \dot{\epsilon}$ :

$$
\operatorname{tr} \mathbf{A}=\frac{3(h(\operatorname{tr} \mathbf{A})-\lambda \dot{\epsilon})}{(h(\operatorname{tr} \mathbf{A})-2 \lambda \dot{\epsilon})(h(\operatorname{tr} \mathbf{A})+\lambda \dot{\epsilon})}
$$

This is a second order equation in terms of $\lambda \dot{\epsilon}$ which can be expressed as

$$
0=2 \operatorname{tr} \mathbf{A}(\lambda \dot{\epsilon})^{2}+(\operatorname{tr} \mathbf{A} h(\operatorname{tr} \mathbf{A})-3)(\lambda \dot{\epsilon})+(3-\operatorname{tr} \mathbf{A} h(\operatorname{tr} \mathbf{A})) h(\operatorname{tr} \mathbf{A})
$$

and has the positive solution

$$
\lambda \dot{\epsilon}=\frac{1}{4 \operatorname{tr} \mathbf{A}}[-(\operatorname{tr} \mathbf{A} h(\operatorname{tr} \mathbf{A})-3)+\sqrt{(\operatorname{tr} \mathbf{A} h(\operatorname{tr} \mathbf{A})-3)(9 \operatorname{tr} \mathbf{A} h(\operatorname{tr} \mathbf{A})-3)}]
$$

In conditions near fully stretched polymers we have $\operatorname{tr} \mathbf{A} h(\operatorname{tr} \mathbf{A}) \gg 3$. Hence, we obtain the asymptotic behaviour

$$
\lambda \dot{\epsilon} \sim \frac{h(\operatorname{tr} \mathbf{A})}{2}
$$

The viscosity in uni-axial elongation is defined by

$$
\bar{\eta}=-\frac{\tau_{z z}-\tau_{r r}}{\dot{\epsilon}}
$$

Inserting the expressions for solvent and polymer stresses in terms of $\dot{\epsilon}, A_{r r}$ and $A_{z z}$ we obtain

$$
\bar{\eta}=3 \eta_{s}+\frac{G h(\operatorname{tr} \mathbf{A})\left(A_{z z}-A_{r r}\right)}{\dot{\epsilon}}=3 \eta_{s}+G \lambda \frac{\operatorname{tr} \mathbf{A} h(\operatorname{tr} \mathbf{A})}{h(\operatorname{tr} \mathbf{A})-\lambda \dot{\epsilon}} \sim 3 \eta_{s}\left[1+\frac{2}{3} c b\left(1-\frac{1}{2 \lambda \dot{\epsilon}}\right)\right](
$$

Here, we have inserted Eq. (75) for nearly fully stretched polymers. 


\section{Appendix II: Maximum Weissenberg number estimate.}

We seek an estimate for the maximum instantaneous Weissenberg number defined below:

$$
W i=\frac{\lambda}{L} \frac{\mathrm{d} L}{\mathrm{~d} t}=\frac{D e}{\zeta} \frac{\mathrm{d} \zeta}{\mathrm{d} \tau}
$$

In Fig. 21 we compare calculated values of the $W i$-number with the asymptote for a freely falling mass. We observe that the predictions of the uniform filament model is very similar to values obtained with the Lagrangian model. The analysis is, therefore, continued based on the governing equations for the uniform filament model. At the time of maximum $W i$-number the following must apply:

$$
0=\frac{\mathrm{d} W i}{\mathrm{~d} \tau}=\frac{\mathrm{d}}{\mathrm{d} \tau}\left(D e \frac{1}{\zeta} \frac{\mathrm{d} \zeta}{\mathrm{d} \tau}\right)=D e\left[-\frac{1}{\zeta^{2}}\left(\frac{\mathrm{d} \zeta}{\mathrm{d} \tau}\right)^{2}+\frac{1}{\zeta} \frac{\mathrm{d}^{2} \zeta}{\mathrm{d} \tau^{2}}\right]
$$

From this equation we find the maximum $W i$-number as follows:

$$
W i_{\max }^{2}=\left(D e \frac{1}{\zeta} \frac{\mathrm{d} \zeta}{\mathrm{d} \tau}\right)_{0}^{2}=\frac{D e^{2}}{\zeta_{0}}\left(\frac{\mathrm{d}^{2} \zeta}{\mathrm{d} \tau^{2}}\right)_{0}
$$

Inserting equation (57) from the uniform filament model we obtain:

$$
\begin{aligned}
W i_{\text {max }}^{2} & =\frac{D e^{2}}{\zeta_{0}}\left\{1-\frac{V}{\zeta_{0}}\left[\left(\frac{1}{\zeta} \frac{\mathrm{d} \zeta}{\mathrm{d} \tau}\right)_{0}+h(\operatorname{tr}(\boldsymbol{A}))_{0}\left(A_{z z}-A_{r r}\right)_{0} \frac{c}{3 D e}\right]\right\} \\
& =\frac{D e}{\zeta_{0}}\left\{D e-\frac{V}{\zeta_{0}}\left[W i_{\text {max }}+h(\operatorname{tr}(\boldsymbol{A}))_{0}\left(A_{z z}-A_{r r}\right)_{0} \frac{c}{3}\right]\right\}
\end{aligned}
$$

This is a quadratic equation in $W i_{\max }$ with the positive solution:

$$
W i_{\text {max }}=\frac{D e V}{2 \zeta_{0}^{2}}\left\{-1+\sqrt{1+\frac{4 \zeta_{0}}{V^{2}}\left(1-\frac{c V}{3 D e \zeta_{0}} h(\operatorname{tr}(\boldsymbol{A}))_{0}\left(A_{z z}-A_{r r}\right)_{0}\right)}\right\}
$$

This is a general result for the uniform filament model. At the time of maximum $W i$ number we assume that elastic effects are not yet important so that the $h(\operatorname{tr} \mathbf{A})$ term in eqauation (83) is negligible. Hence,

$$
W i_{\text {max }}=\frac{D e V}{2 \zeta_{0}^{2}}\left\{-1+\sqrt{1+\frac{4 \zeta_{0}}{V^{2}}}\right\}
$$

This is a decreasing function in $\zeta_{0}$ and an upper limit for $W i_{\max }$ is found for $\zeta_{0}=\zeta(0)=\Lambda$ :

$$
W i_{\max }=\frac{D e V}{2 \Lambda^{2}}\left\{-1+\sqrt{1+\frac{4 \Lambda}{V^{2}}}\right\}
$$

Furthermore, we note that as $V \rightarrow 0$ we have $W i_{\text {max }} \rightarrow D e / \zeta_{0}^{3 / 2} \leq D e / \Lambda^{3 / 2}$. 


\section{References}

[1] S. L. Anna and G. H. McKinley. Elasto-capillary thinning and breakup of model elastic liquids. Journal of Rheology, 45:115-138, 2001.

[2] S. L. Anna, G. H. McKinley, D. A. Nguyen, T. Sridhar, S. J. Muller, J. Huang and D. F. James. An interlaboratory comparison of measurements from filamentstretching rheometers using common test fluids. Journal of Rheology, 45:83-114, 2001.

[3] A. Bach, H. K. Rasmussen, and O. Hassager. Extensional viscosity for polymer melts measured in the filament stretching rheometer. Journal of Rheology, 47:429441, 2003.

[4] R. B. Bird, R. C. Armstrong, and O. Hassager. Dynamics of Polymeric Liquids. Volume 1: Fluid Mechanics. John Wiley \& Sons, New York, 2nd edition, 1987.

[5] H. Chang and A. S. Lodge. A possible mechanism for stabilizing elongational flow in certain polymeric liquids at constant temperature and composition. Rheologica Acta, 10:448-449, 1971.

[6] L. Campo-Deano and C. Clasen. The Slow Retraction Method (SRM) for the determination of ultra-short relaxation times in capillary breakup experiments. Journal of Non-Newtonian Fluid Mechanics, 165:1688-1699, 2010.

[7] C. Clasen. Capillary Breakup Extensional Rheometry of Semi-dilute Polymer Solutions. Korea-Australia Rheology Journal, 22:331-338, 2010.

[8] C. Clasen, J. Bico, V. M. Entov and G. H. McKinley. 'Gobbling drops': the jettingdripping transition in flows of polymer solutions. Journal of Fluid Mechanics, 636:540, 2009.

[9] C. Clasen, J. Eggers, M. A. Fontelos, J. Li and G. H. McKinley. The beads-on-string structure of viscoelastic threads. Journal of Fluid Mechanics, 556:283-308, 2006.

[10] C. Clasen, J. P. Plog, W. M. Kulicke, M. Owens, C. Macosko, L. E. Scriven, M. Verani and G. H. McKinley. How dilute are dilute solutions in extensional flows? Journal of Rheology, 50:849-881, 2006.

[11] J. Eggers. Nonlinear dynamics and breakup of free-surface flows. Reviews of Modern Physics, 69:865-929, 1997.

[12] V. M. Entov and E. J. Hinch. Effect of a spectrum of relaxation times on the capillary thinning of a filament of elastic liquid. Journal of Non-Newtonian Fluid Mechanics, 72:31-53, 1997.

[13] I. Ghosh, G. H. McKinley, R. A. Brown and R. C. Armstrong. Deficiencies of FENE dumbbell models in describing the rapid stretching of dilute polymer solutions. Journal of Rheology, 45:721-758, 2001.

[14] O. G. Harlen, J. M. Rallison and P. Szabo. A split Lagrangian-Eulerian method for simulating transient viscoelastic flows. Journal of Non-Newtonian Fluid Mechanics, 60:81-104, 1995. 
[15] O. Hassager, J. M. R. Marin, K. J. Yu and H. K. Rasmussen. Polymeric liquids in extension: Fluid mechanics or rheometry? Rheologica Acta, 49:543-554, 2010.

[16] C. C. Hsieh and R. Larson. Prediction of coil-stretch hysteresis for dilute polystyrene molecules in extensional flow. Journal of Rheology, 49:1081-1089, 2005.

[17] W. M. Jones, N. E. Hudson and J. Ferguson. The extensional properties of M1 obtained from the stretching of a filament by a falling pendant drop. Journal of Non-Newtonian Fluid Mechanics, 35:263-276, 1990.

[18] W. M. Jones and I. J. Rees. The stringiness of dilute polymer solutions. Journal of Non-Newtonian Fluid Mechanics, 11:257-268, 1982.

[19] R. A. Keiller. Extending filaments of an Oldroyd-B fluid. Journal of Non-Newtonian Fluid Mechanics, 42:37-48, 1992.

[20] R. Keunings. On the Peterlin approximation for finitely extensible dumbbells. Journal of Non-Newtonian Fluid Mechanics, 68:85-100, 1997.

[21] M. I. Kolte and P. Szabo. Capillary thinning of polymeric filaments. Journal of Rheology, 43:609-625, 1999.

[22] L. D. Landau and E. M. Lifshitz. Fluid Mechanics. Pergamon, Oxford, 1987.

[23] L. Li, R. G. Larson and T. Sridhar. Brownian dynamics simulations of dilute polystyrene solutions. Journal of Rheology, 44:291-322, 2000.

[24] R. F. Liang and M. R. Mackley. Rheological characterization of the time and strain dependence for polyisobutylene solutions. Journal of Non-Newtonian Fluid Mechanics, 52:387-405, 1994.

[25] A. Lindner, J. Vermant and D. Bonn. How to obtain the elongational viscosity of dilute polymer solutions? Physica A-Statistical Mechanics and Its Applications, 319:125-133, 2003.

[26] M. E. Mackay and D. V. Boger. An explanation of the rheological properties of Boger fluids. Journal of Non-Newtonian Fluid Mechanics, 22:235-243, 1987.

[27] J. M. R. Marin and H. K. Rasmussen. Lagrangian finite-element method for the simulation of K-BKZ fluids with third order accuracy. Journal of Non-Newtonian Fluid Mechanics, 156:177-188, 2009.

[28] J. E. Matta and R. P. Tytus. Liquid stretching using a falling cylinder. Journal of Non-Newtonian Fluid Mechanics, 35:215-229, 1990.

[29] G. H. McKinley and T. Sridhar. Filament-stretching rheometry of complex fluids. Annual Review of Fluid Mechanics, 34:375-415, 2002.

[30] G. H. McKinley and A. Tripathi. How to extract the Newtonian viscosity from capillary breakup measurements in a filament rheometer. Journal of Rheology, 44:653-670, 2000 . 
[31] E. Miller, C. Clasen and J. P. Rothstein. The effect of step-stretch parameters on capillary breakup extensional rheology (CaBER) measurements. Rheologica Acta, 48:625-639, 2009.

[32] J. Meissner. Experimental aspects in polymer melt elongational rheometry. Chemical Engineering Communications, 33:159-180, 1985.

[33] N. Morrison and J. M. Rallison. Transient 3D-flow of polymer solutions: A Lagrangian computational method. Journal of Non-Newtonian Fluid Mechanics, 165: 1241-1257, 2010.

[34] H. Münstedt. New universal extensional rheometer for polymer melts - measurements on a polystyrene sample. Journal of Rheology, 23:421-436, 1979.

[35] H. Münstedt, S. Kurzbeck and L. Egersdörfer. Influence of molecular structure on rheological properties of polyethylenes. ii. elongational behavior. Rheologica Acta, 37:21-29, 1998.

[36] J. P. Plog, W. M. Kulicke and C. Clasen. Influence of the molar mass distribution on the elongational behaviour of polymer solutions in capillary breakup. Applied Rheology, 15:28-37, 2005.

[37] H. K. Rasmussen. Time-dependent finite-element method for the simulation of threedimensional viscoelastic flow with integral models. Journal of Non-Newtonian Fluid Mechanics, 84:217-232, 1999.

[38] H. K. Rasmussen and O. Hassager. Simulation of transient viscoelastic flow with second order time integration. Journal of Non-Newtonian Fluid Mechanics, 56:6584, 1995.

[39] M. Renardy. Some comments on the surface-tension driven break-up (or the lack of it) of viscoelastic jets. Journal of Non-Newtonian Fluid Mechanics, 51:97-107, 1994.

[40] M. Renardy. A numerical study of the asymptotic evolution and breakup of Newtonian and viscoelastic jets. Journal of Non-Newtonian Fluid Mechanics, 59:267-282, 1995.

[41] M. Sentmanat, B. N. Wang and G. H. McKinley. Measuring the transient extensional rheology of polyethylene melts using the SER universal testing platform. Journal of Rheology, 49:585-606, 2005.

[42] L. B. Smolka and A. Belmonte. Drop pinch-off and filament dynamics of wormlike micellar fluids. Journal of Non-Newtonian Fluid Mechanics, 115:1-25, 2003.

[43] L. B. Smolka, A. Belmonte, D. M. Henderson and T. P. Witelski. Exact solution for the extensional flow of a viscoelastic filament. European Journal of Applied Mathematics, 15:679-712, 2004.

[44] S. H. Spiegelberg, D. C. Ables and G. H. McKinley. The role of end-effects on measurements of extensional viscosity in filament stretching rheometers. Journal of Non-Newtonian Fluid Mechanics, 64:229-267, 1996. 
[45] T. Sridhar, V. Tirtaatmadja, D. A. Nguyen and R. K. Gupta. Measurement of extensional viscosity of polymer solutions. Journal of Non-Newtonian Fluid Mechanics, 40:271-280, 1991.

[46] M. Stelter and G. Brenn. Validation and application of a novel elongational device for polymer solutions. Journal of Rheology, 44:595-616, 2000.

[47] Y. M. Stokes, E. O. Tuck and L. W. Schwartz. Extensional fall of a very viscous fluid drop. Quarterly Journal of Mechanics and Applied Mathematics, 53:565-582, 2000.

[48] P. Szabo. Transient filament stretching rheometer I: force balance analysis. Rheologica Acta, 36:277-284, 1997.

[49] P. Szabo and G. H. McKinley. Filament stretching rheometer: inertia compensation revisited. Rheologica Acta, 42:269-272, 2003.

[50] M. Yao and G. H. McKinley. Numerical simulation of extensional deformations of viscoelastic liquid bridges in filament stretching devices. Journal of Non-Newtonian Fluid Mechanics, 74:47-88, 1998.

[51] M. Yao, G. H. McKinley and B. Debbaut. Extensional deformation, stress relaxation and necking failure of viscoelastic filaments. Journal of Non-Newtonian Fluid Mechanics, 79:469-501, 1998. 


\section{Figure captions}

Figure 1: Sequence of images of a polystyrene solution confined between two circular plates of radius $R_{0}=5.12 \mathrm{~mm}$, stretched by a falling mass $(m=5.8155 \mathrm{~g}$ ) that exerts a constant force pull on the elongating filament over a distance of $L_{\max }=1.3 \mathrm{~m}$. Time between consecutive images is $\Delta t=45 \mathrm{~ms}$. The initial aspect ratio of the cylindrical fluid sample is $\Lambda=L_{0} / R_{0}=0.4937$. The first four images show the downwards accelerating stage used to initially hold and release the falling mass. The left hand vertical dashed line depicts the point between images where the polymer stretch $A$ approaches its finite extensibility limit $b$. The second vertical broken line indicates the limit up to which the radius at the axial mid-point $L(t) / 2$ can still be directly observed.

Figure 2: Definition sketch for a constant force pull (CFP) experiment. A liquid bridge is initially suspended between a fixed upper plate and a bottom plate assembly having a mass $m$. At time $t=0$ the bottom plate is instantaneously released and falls freely due to gravity.

Figure 3: Constant force stretching of a Boger fluid (polystyrene with $M_{w}=2.84 \times 10^{6}$ $\mathrm{g} / \mathrm{mol}$ dissolved in styrene oligomers at a concentration of $0.025 \mathrm{wt} \%$ ). The minimum filament diameter $2 R_{\text {mid }}$ versus time is given for small aspect ratios $0.16 \leq \Lambda \leq 0.23$ and different attached masses $m$.

Figure 4: Constant force stretching of a Boger fluid (polystyrene with $M_{w}=2.84 \times 10^{6}$ $\mathrm{g} / \mathrm{mol}$ dissolved in styrene oligomers at a concentration of $0.025 \mathrm{wt} \%$ ). The minimum filament diameter $2 R_{\text {mid }}$ versus time is given for medium aspect ratios $0.23 \leq \Lambda \leq 0.32$ and different attached masses $m$.

Figure 5: Constant force stretching of a Boger fluid (polystyrene with $M_{w}=2.84 \times 10^{6}$ $\mathrm{g} / \mathrm{mol}$ dissolved in styrene oligomers at a concentration of $0.025 \mathrm{wt} \%$ ). The minimum filament diameter $2 R_{\text {mid }}$ versus time is given for large aspect ratios $\Lambda \geq 0.4$ and different attached masses $m$.

Figure 6: Acceleration $\left(d^{2} L_{a p p} / d t^{2}\right) / g$ of the falling weight from the medium aspect ratio experiments of Figure 4 as a function of the Hencky strain $\ln \left(L_{a p p} / L_{0}\right)$. The apparent length $L_{a p p}$ is determined from the radii $R_{\text {mid }}$ of Figure 4 assuming a cylindrical filament shape, $L_{\text {app }}=L_{0} \pi R_{0}^{2} / \pi R_{\text {mid }}^{2}$.

Figure 7: Acceleration $\left(d^{2} L_{a p p} / d t^{2}\right) / g$ of a falling weight of $m \sim 1.8 \mathrm{~g}$ for different initial aspect ratios as a function of the Hencky strain $\ln \left(L_{a p p} / L_{0}\right)$. The apparent length $L_{a p p}$ is determined from the radii data $R_{m i d}$ from Figure 3 - 5 assuming a cylindrical filament shape, $L_{\text {app }}=L_{0} \pi R_{0}^{2} / \pi R_{m i d}^{2}$.

Figure 8: The shape of the filament surface $R(z, t)$ at a Hencky $\operatorname{strain} \ln \left(L / L_{0}\right)=1.0$. Comparison of experimental data $(+)$ with the lubrication solution (- -) in (29) and a numerical simulation (-) utilising the condition (28). The fluid filament is Newtonian having an initial aspect ratio of $\Lambda=0.5731$.

Figure 9: Comparison of experimental data with numerical simulation of the constant 
force pull for a) the mid-point diameter of an elongating filament, and b) the force measured at the upper endplate with the force $F(t)=m g-m \ddot{L}$ from eq. (25). The Newtonian test fluid is a viscous PDMS oil with $\eta_{s}=61.4 \mathrm{~Pa} \mathrm{~s}$ and $\sigma=0.02 \mathrm{~N} / \mathrm{m}, m=2.8 \mathrm{~g}$. The corresponding dimensionless parameters (defined in Section 4.1) are $V=11.56, \mathrm{Ca}=$ $2064, \Lambda=0.4781$.

Figure 10: Comparison of experimental data of the mid-filament diameter evolution for the polymer solution with numerical simulation for a FENE-P fluid with $b=800, \eta_{s}=$ $86.5 \mathrm{~Pa} \mathrm{~s}, \sigma=0.035 \mathrm{~N} / \mathrm{m}, \lambda=24.8 \mathrm{~s}$ (with the corresponding dimensionless parameters $C a=1551$ and $D e=1085)$ : Test I for $m=20.8 \mathrm{~g}$ with $\Lambda=0.1659(V=0.761)$; and Test II for $m=5.8 \mathrm{~g}$ with $\Lambda=0.2120(V=3.47)$.

Figure 11: Comparison of experimental force data for the polymer solution with numerical simulation: (FENE-P fluid with $b=800, \eta_{s}=86.5 \mathrm{~Pa} \mathrm{~s}, \sigma=0.035 \mathrm{~N} / \mathrm{m}, \lambda=24.8 \mathrm{~s}$ and $m=20.8 \mathrm{~g}$ with $\Lambda=0.1659)$.

Figure 12: Surface shape at $\tau=39.5$ for a Newtonian fluid with $\Lambda=0.4781$ and capillary numbers (eq. (44)) equal to $C a=100,1000,2064$ and 10000 respectively.

Figure 13: Comparison of simulated acceleration for the uniform filament model (eq. (49), solid line) and the pertubation solutions (broken lines). For a Newtonian fluid with $V=0.1$ and $\Lambda=1$ the 1 st order pertubation (dashed line) comes quite close and the 2nd order pertubation cannot be distinguished graphically from the numerical solution of (49). The maximum deviation occurs at $\tau \approx 1$ and is of magnitude $\Delta \ddot{\zeta} / \ddot{\zeta} \approx 0.001$.

Figure 14: Comparison of simulated acceleration and pertubation solution for a large value of $V=1$. For a Newtonian fluid with $V=1.0$ and $\Lambda=1$, the 2nd order pertubation is required for a good approximation to the numerical solution of the uniform filament model (eq. (49)).

Figure 15: a) Evolution in the midplane radius, and b) falling plate acceleration versus dimensionless time in the falling cylinder experiment. FENE-P fluid with $c=0.52$, $b=1000, D e=248, V=1$ and $\Lambda=1$.

Figure 16: a) Acceleration of the falling plate and b) polymer stretch $\operatorname{tr} \mathbf{A}$ versus accumulated Hencky strain at the midplane of the elongating filament. FENE-P fluid with $c=0.52, b=1000, D e=248, V=1$ and $\Lambda=1$.

Figure 17: Acceleration of the falling mass a) versus time, and b) versus Hencky strain (uniform filament model). FENE-P fluid with $c=0.52, b=1000, D e=248$ and $\Lambda=1$ for $1 \leq V \leq 6.6$.

Figure 18: Computed evolution in the polymer stretch $\operatorname{tr} \mathbf{A}$ versus axial strain (uniform filament model). FENE-P fluid with $c=0.52, b=1000, D e=248$ and $\Lambda=1$ for $1 \leq V \leq 6.6$. The asymptotic expression shown by the dash-dotted line is evaluated in section 7 (eq. (62)).

Figure 19: Filament radius versus time (uniform filament model). Different extensibility 
FENE fluids with $c=0.52, D e=248, V=1$ and $\Lambda=1$. The insert shows a close-up of the curves for radii $0.02 \leq R / R_{0} \leq 0.2$.

Figure 20: Evolution in the polymer stretch versus axial strain (uniform filament model) compared with the asymptote $\operatorname{tr} \mathbf{A}=2+(\zeta / \Lambda)^{2}$ of eq. (66). FENE-P fluids with $c=0.52$, $D e=248, V=1, \Lambda=1$ and different $b$-values. The open circles mark the critical strain $\zeta_{b}$ for the respective $b$ values obtained from eq. (67).

Figure 21: The instantaneous Weissenberg number versus time. Comparison of Lagrangian prediction with uniform filament model and the free falling asymptote. FENE-P fluid with $c=0.52, b=1000, V=1$ and $\Lambda=1$. 
Figure(s)

$t<0 \quad t>0$
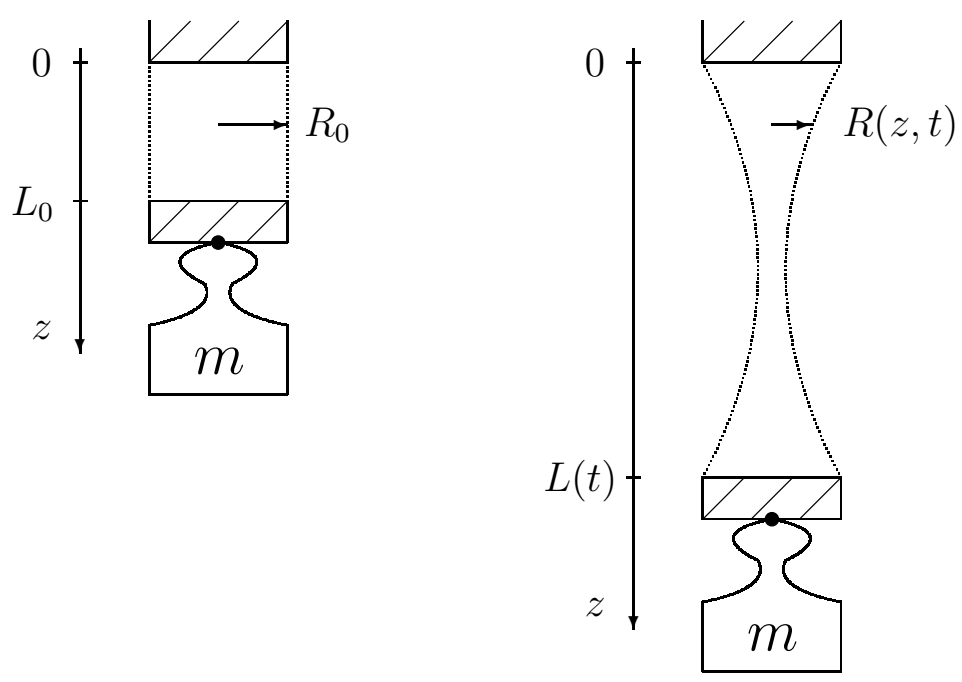

Figure 2: 


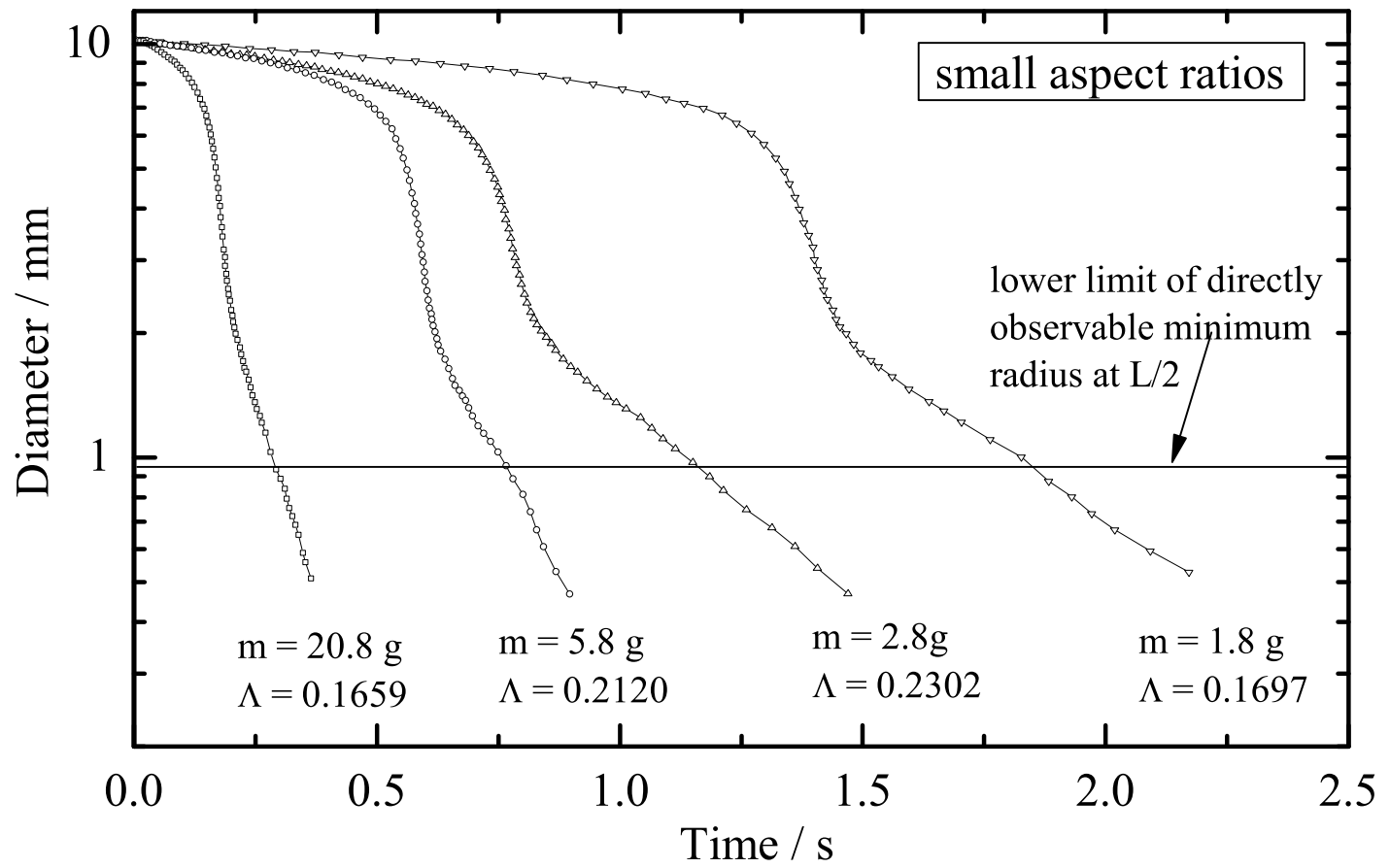

Figure 3: 


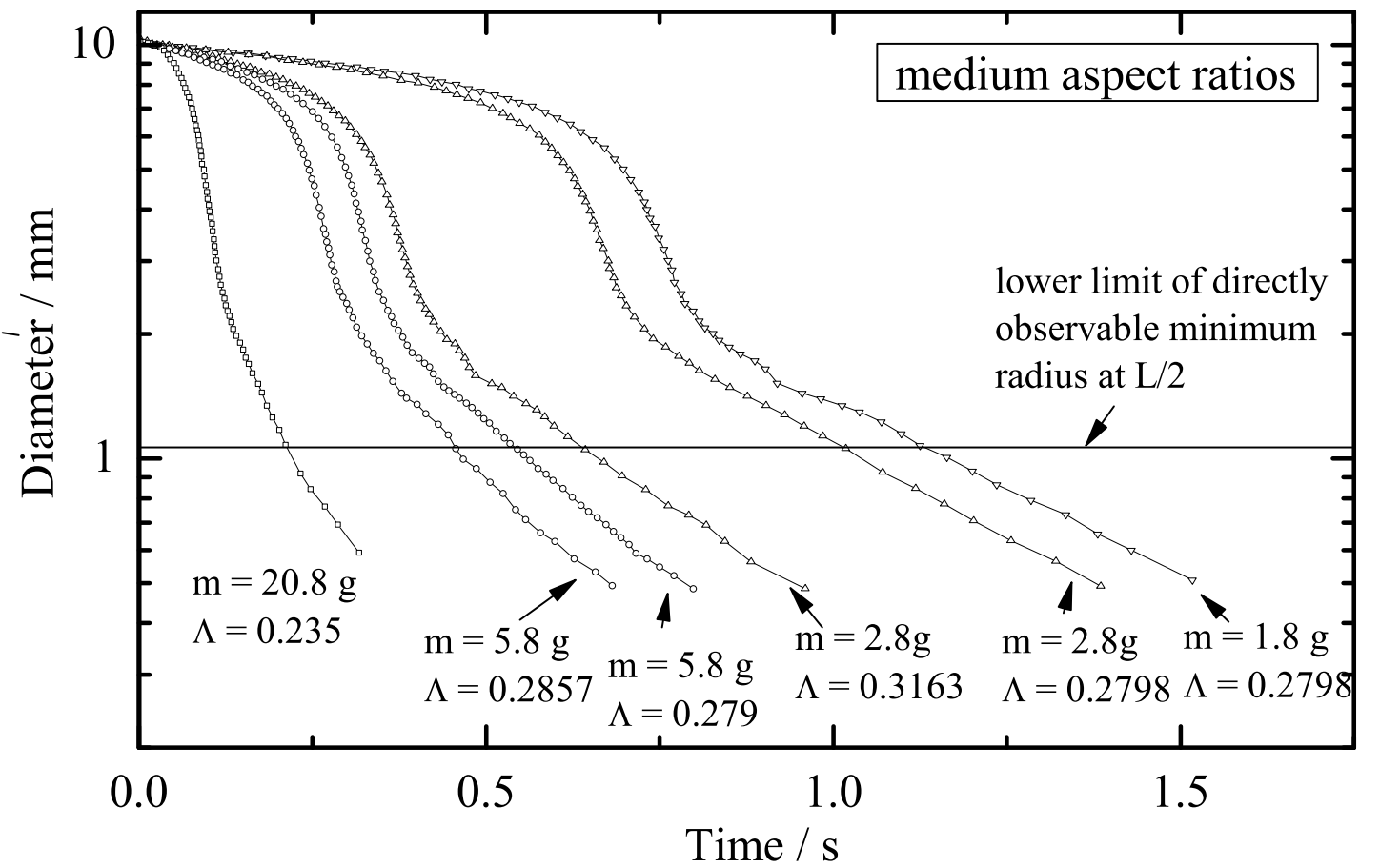

Figure 4: 
Figure(s)

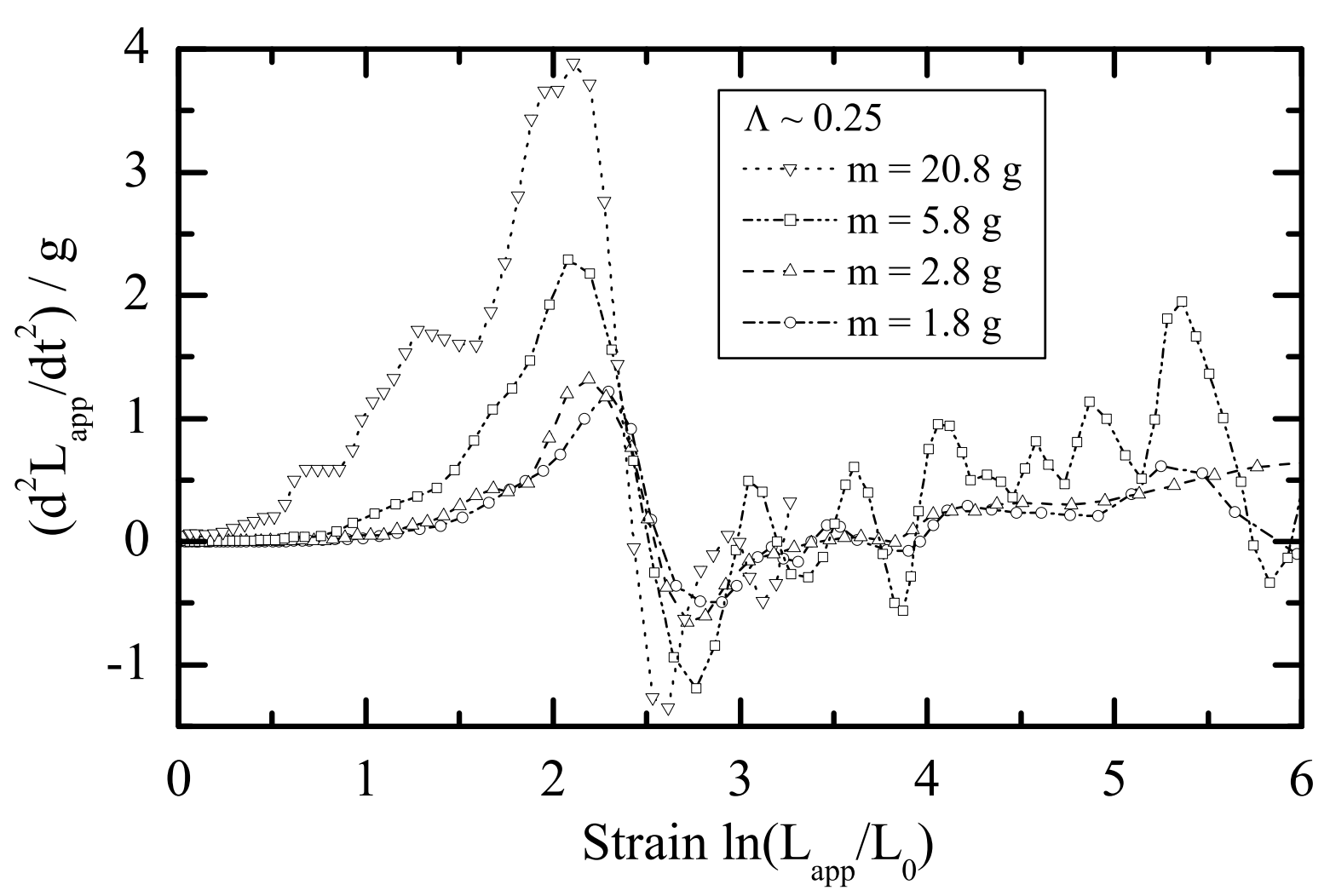

Figure 6: 
Figure(s)

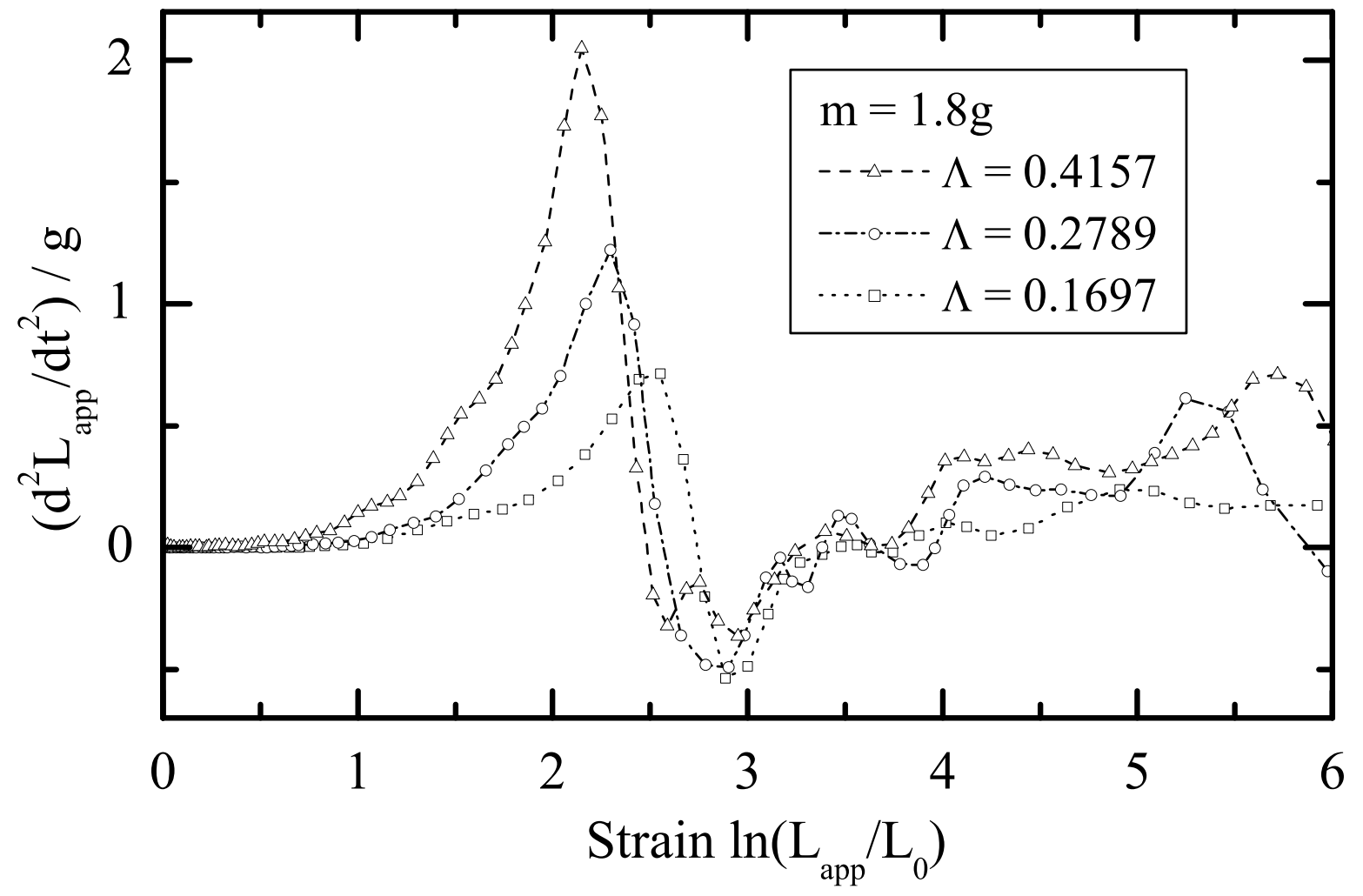

Figure 7: 
Figure(s)

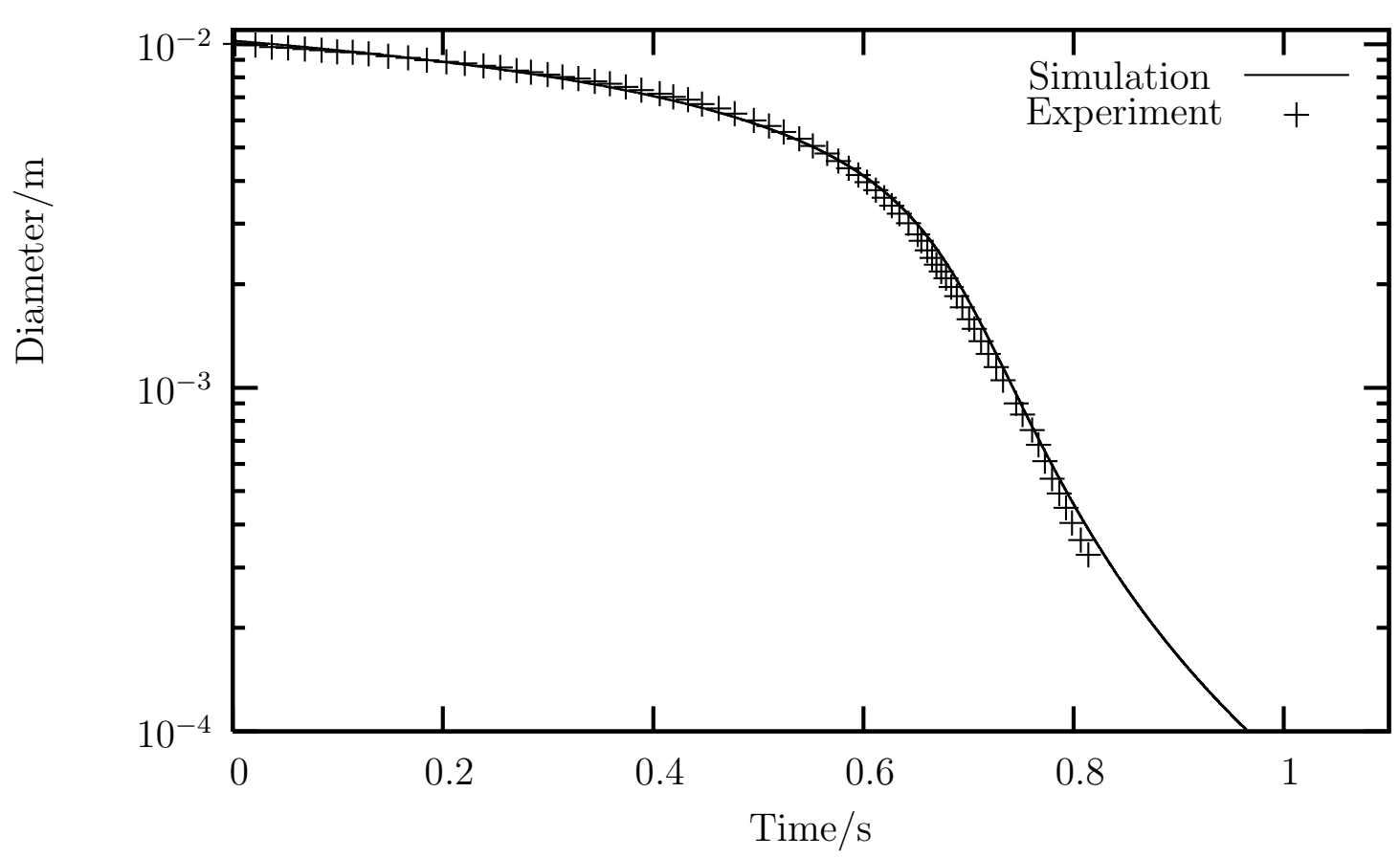

Figure 9: a 
Figure(s)

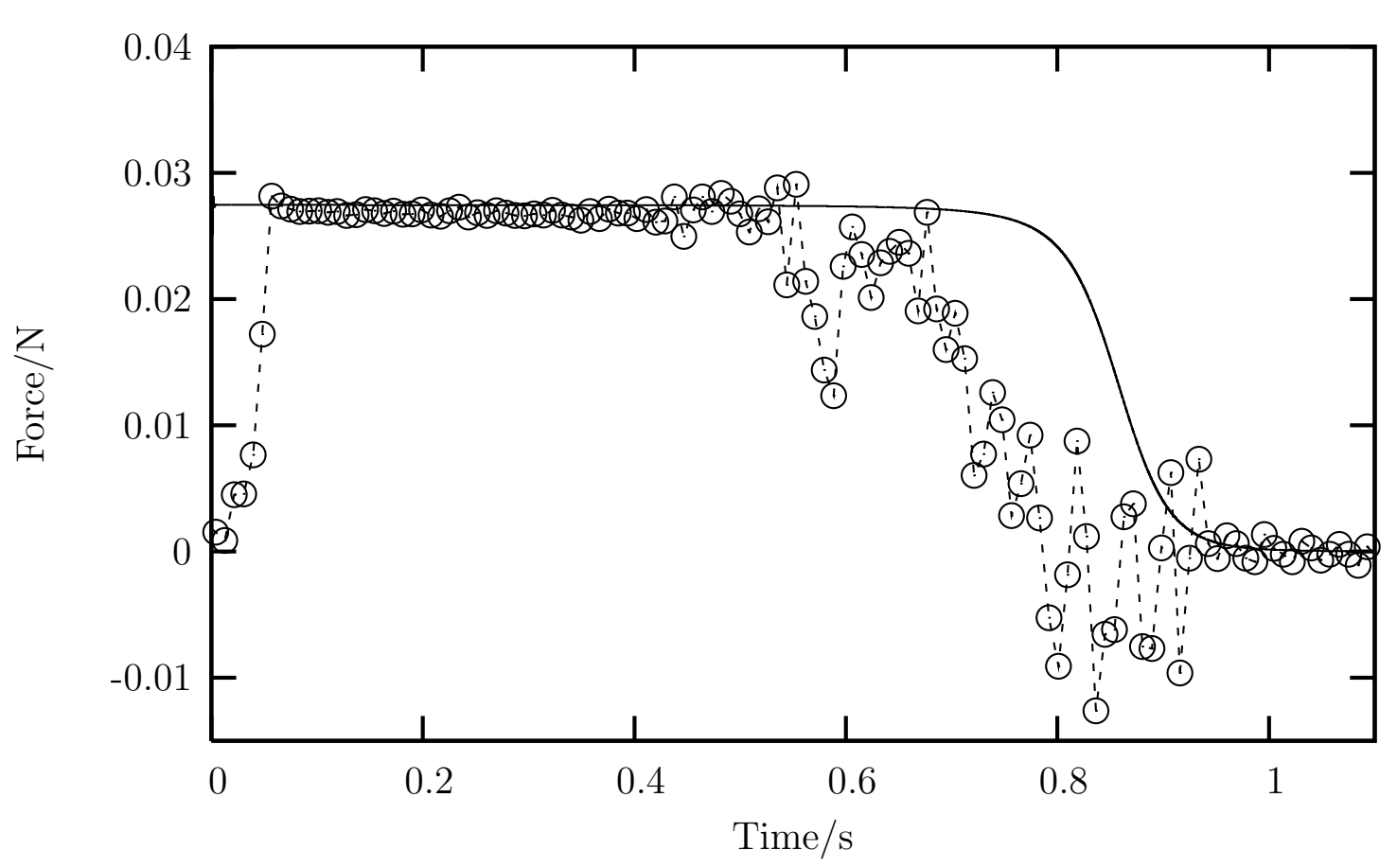

Figure 9: b 
Figure(s)

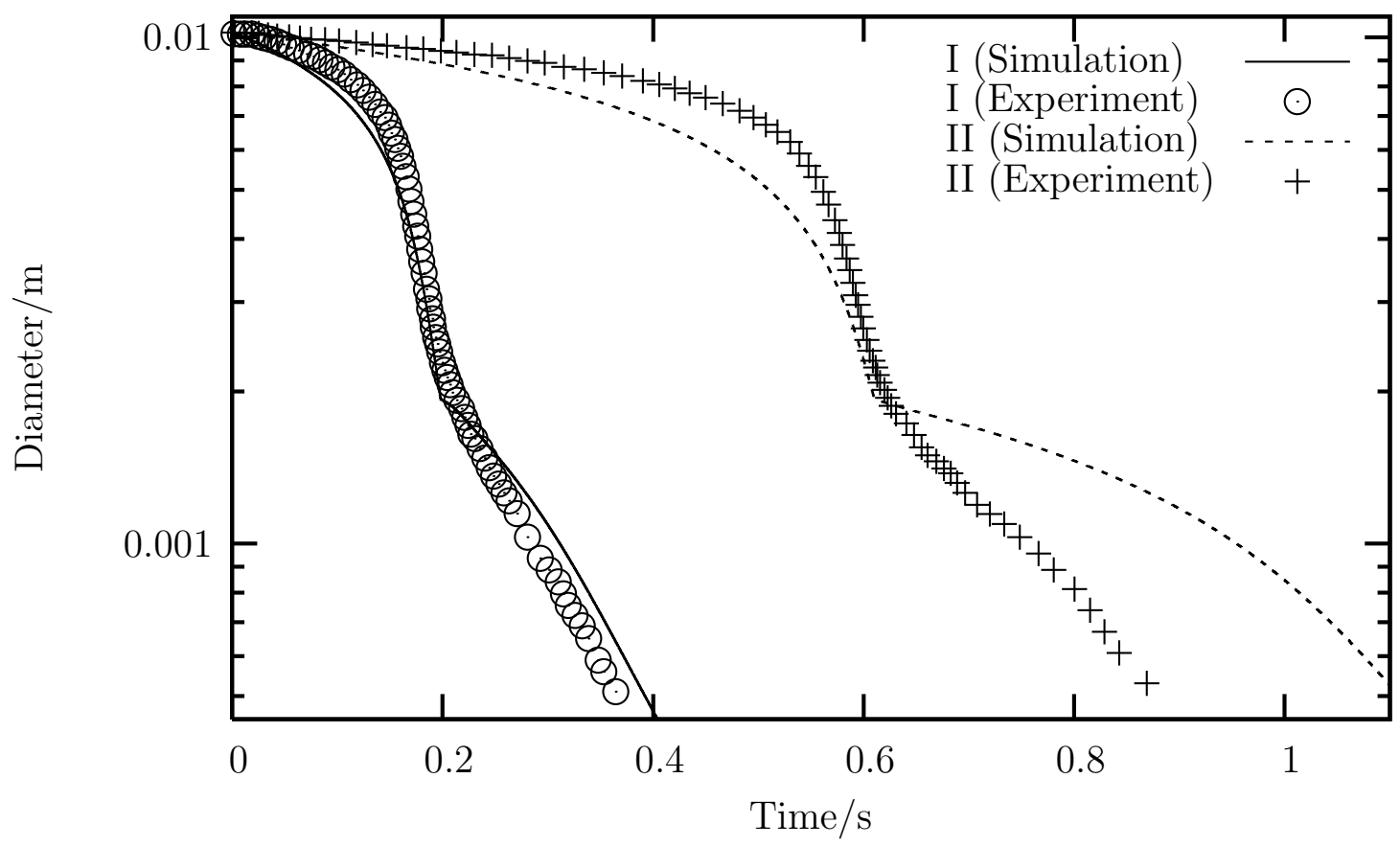

Figure 10: 
Figure(s)

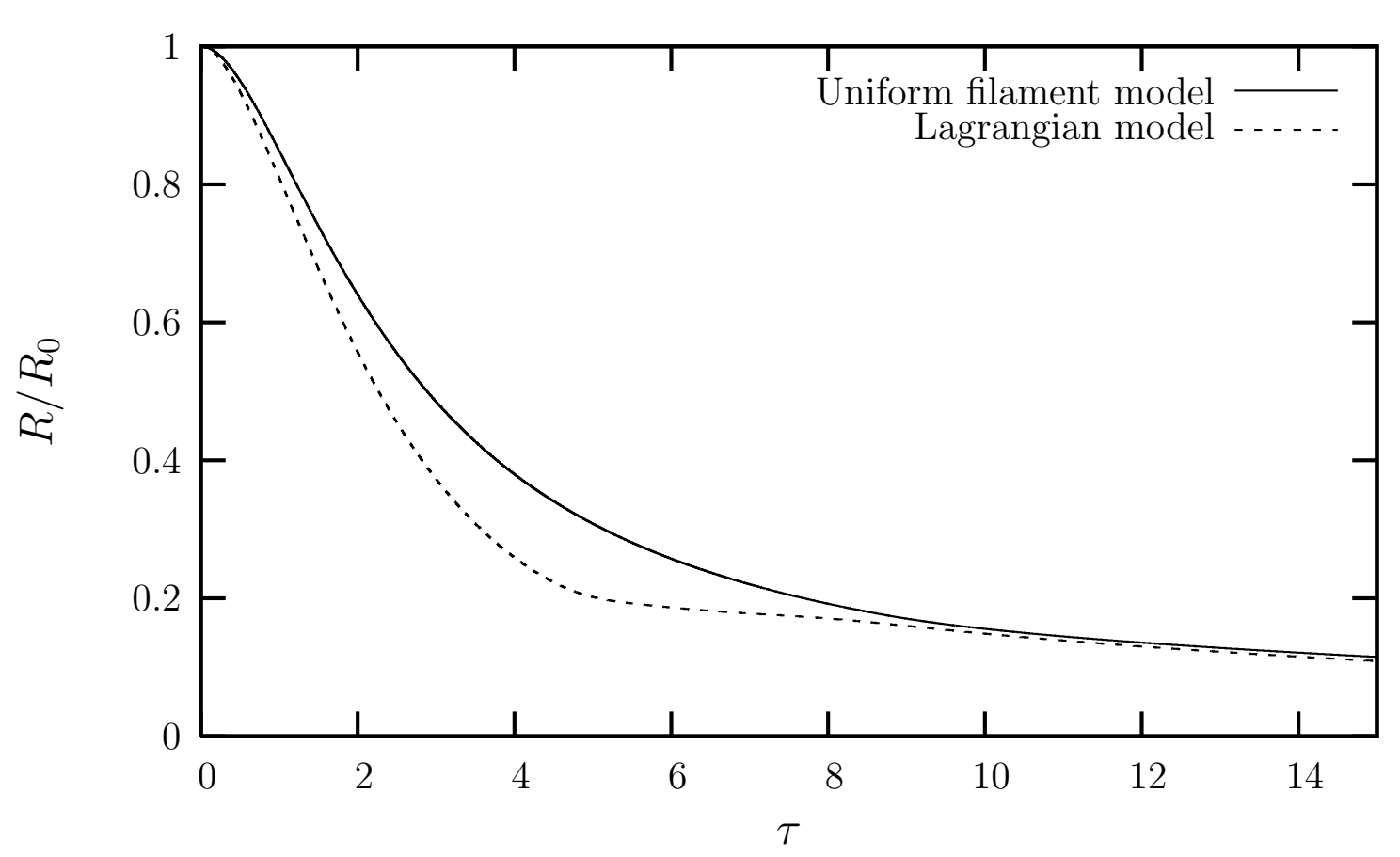

Figure 15: a 
Figure(s)

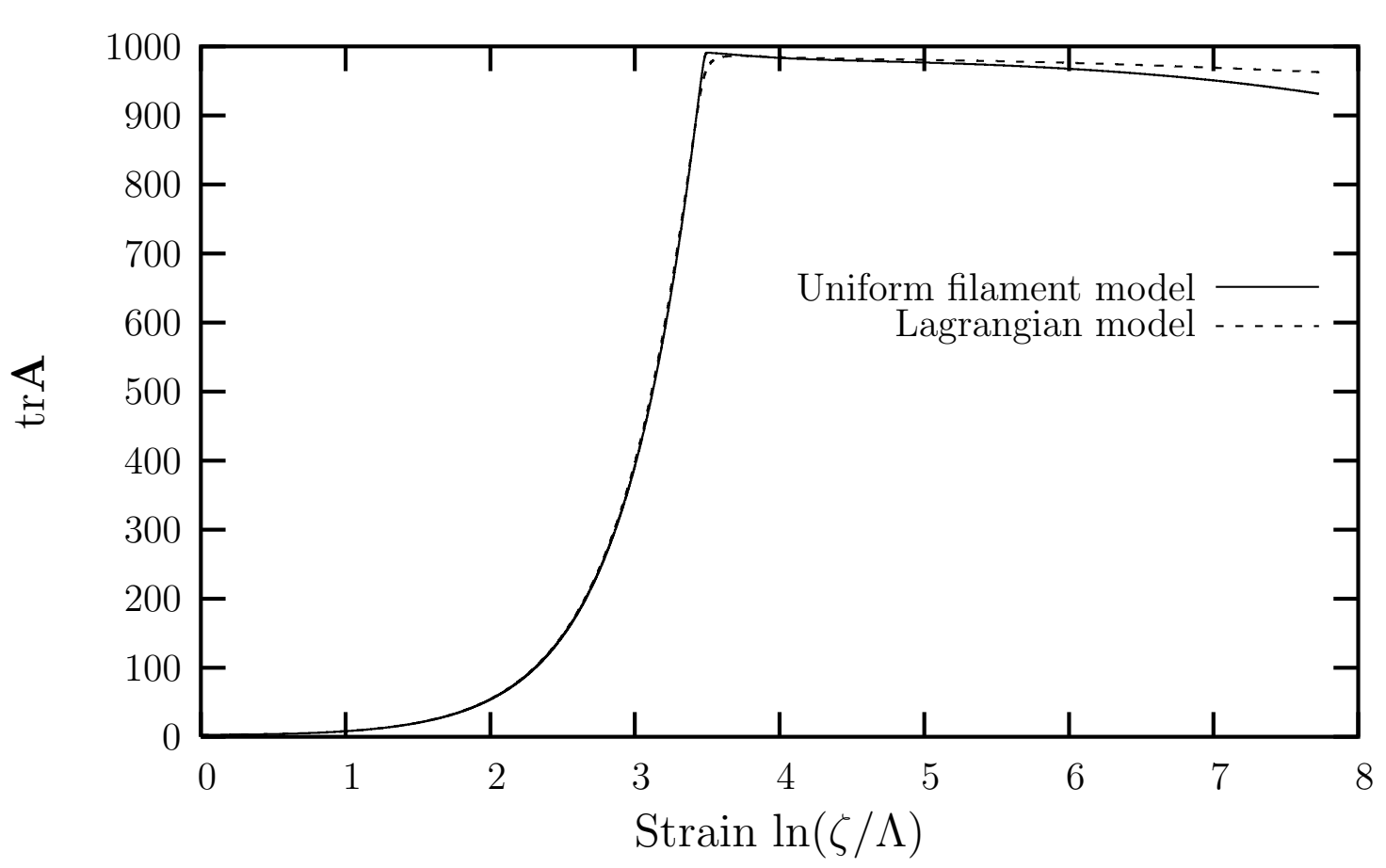

Figure 16: b 
Figure(s)

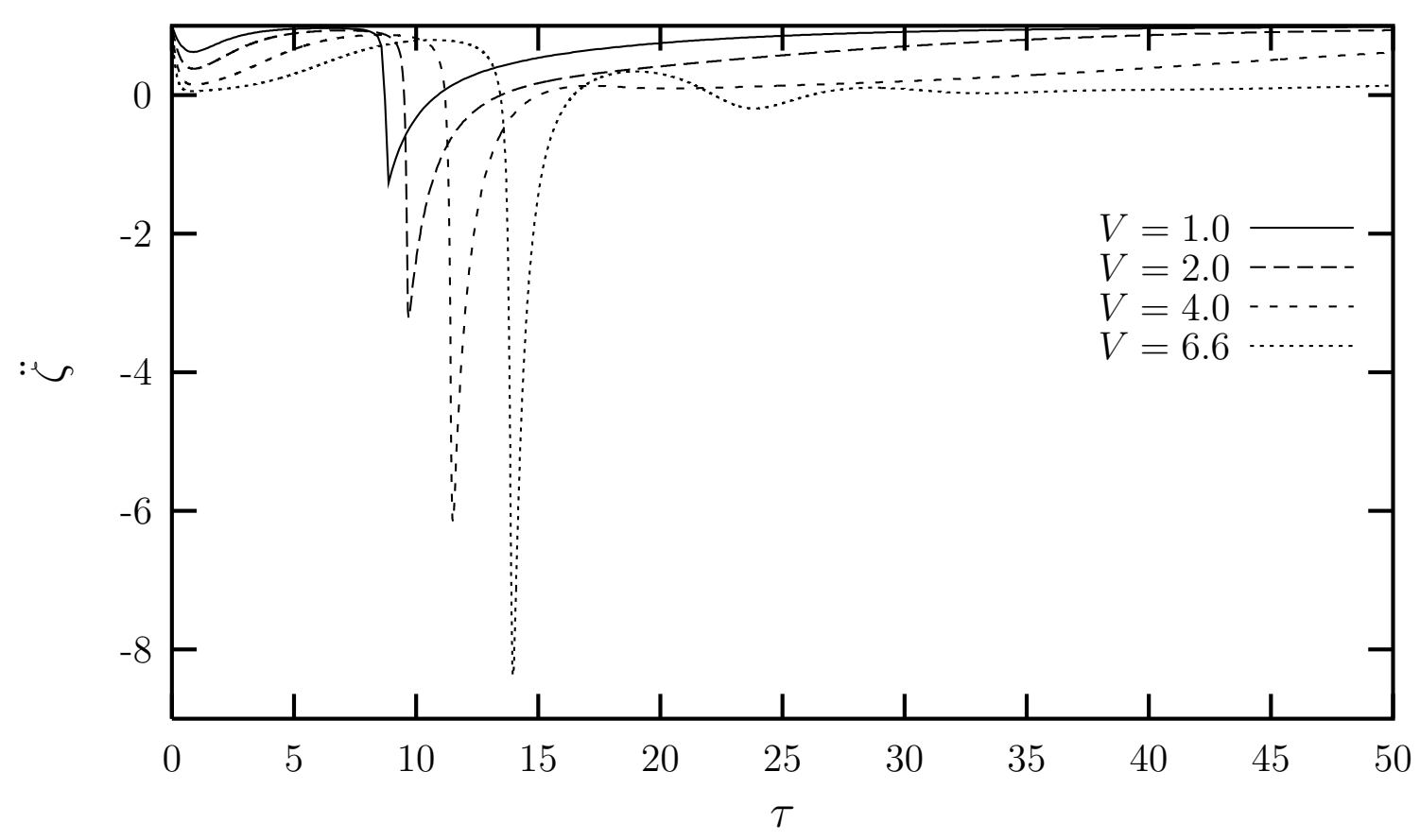

Figure 17: a 
Figure(s)

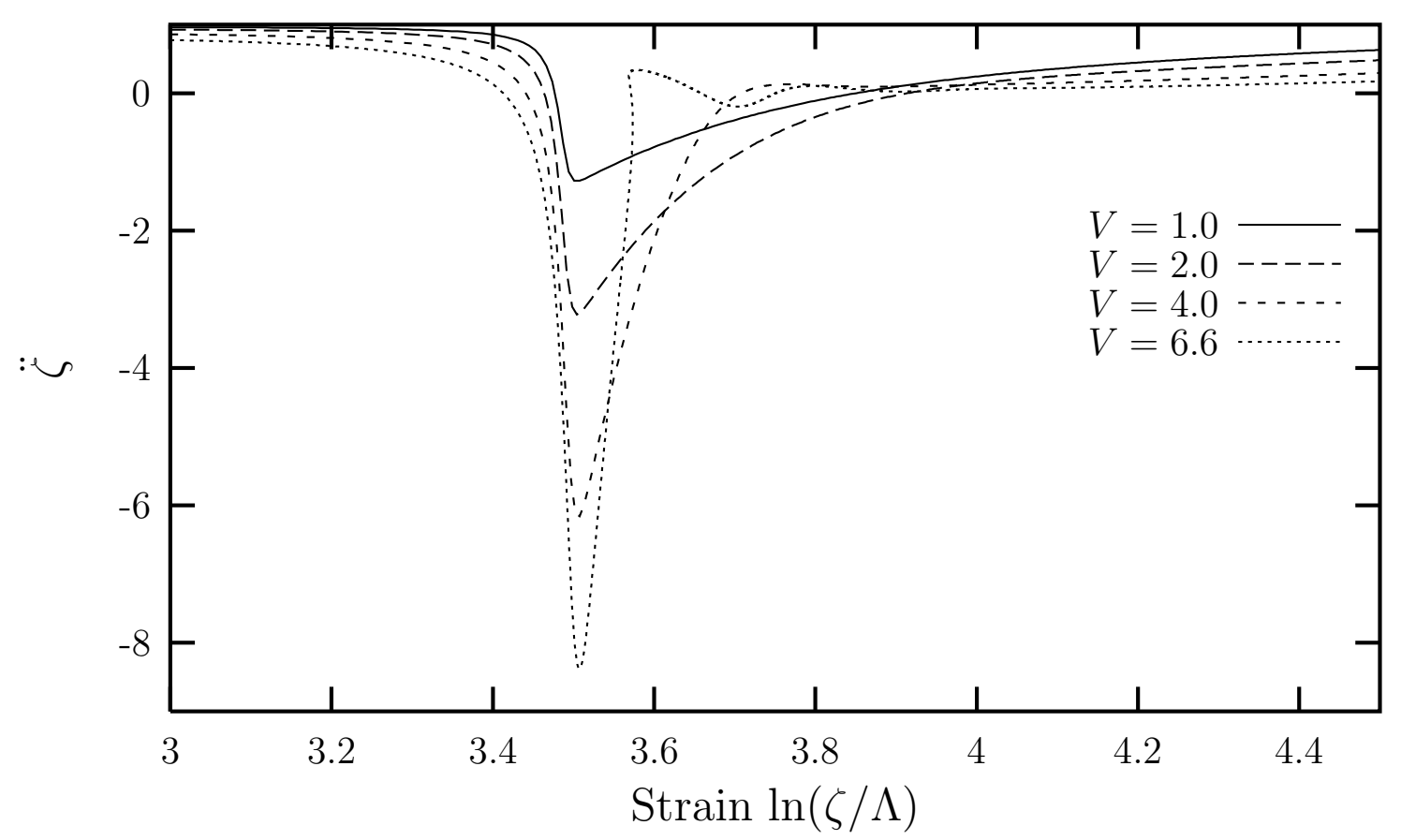

Figure 17: b 


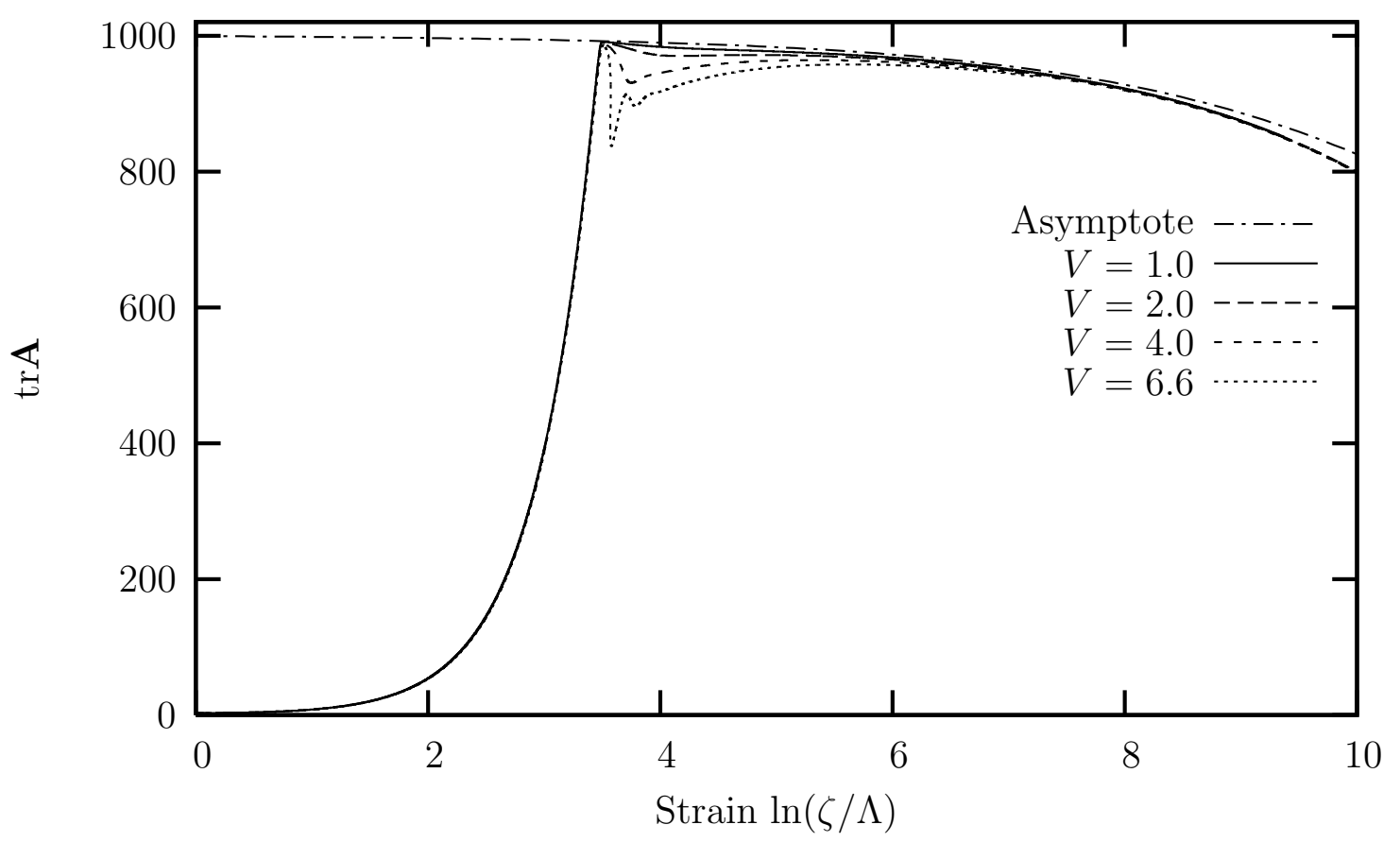

Figure 18: 
Figure(s)

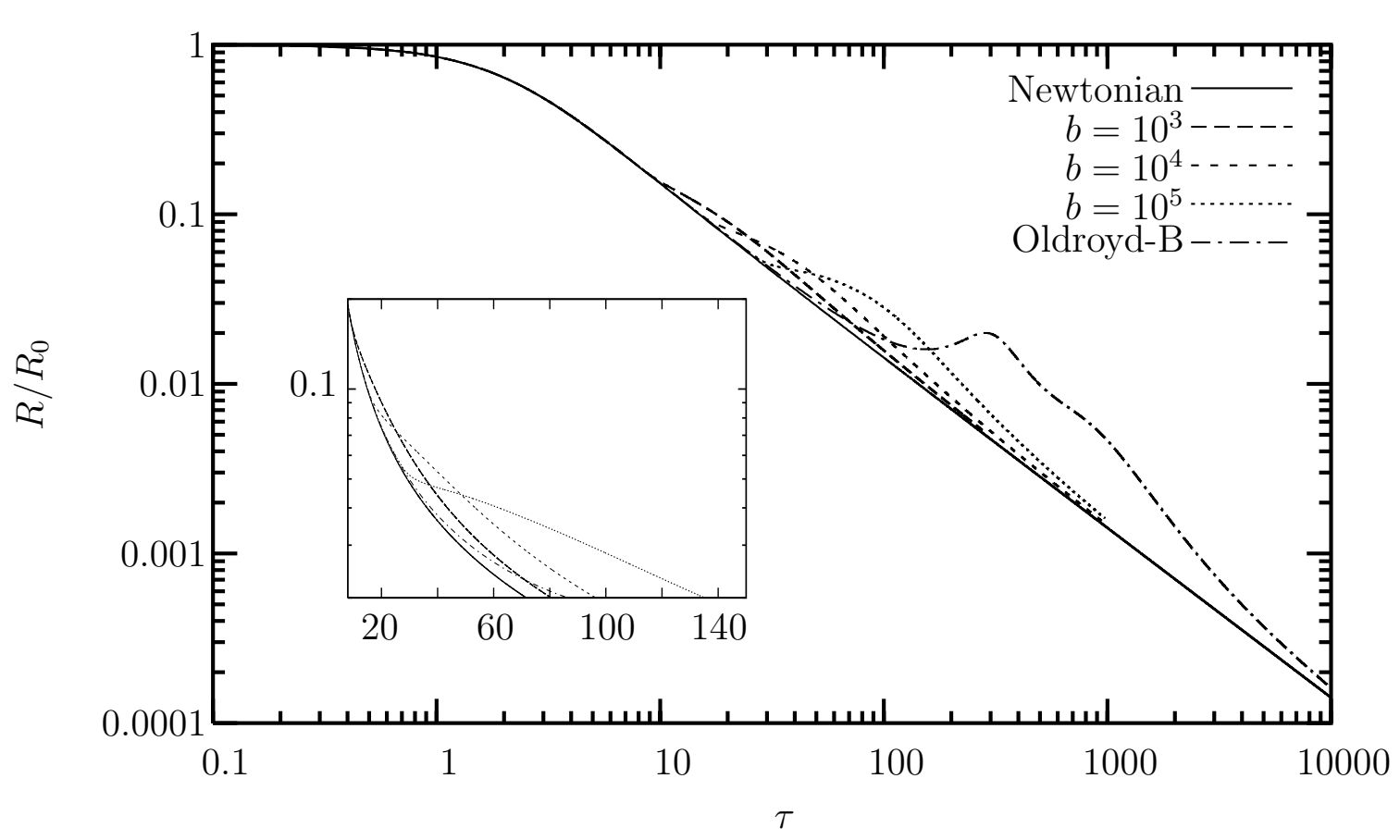

Figure 19: 
Figure(s)

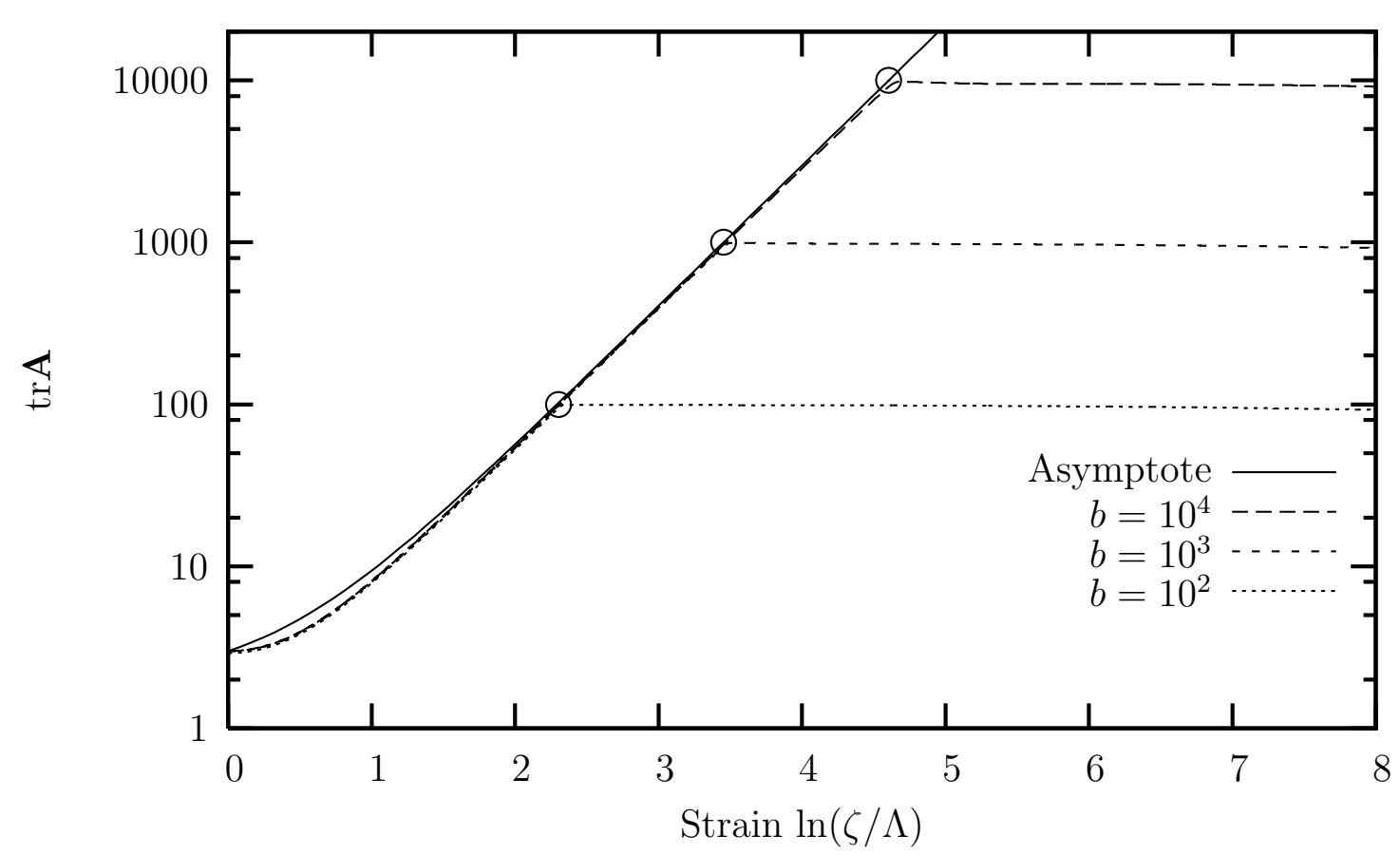

Figure 20: 
Figure(s)

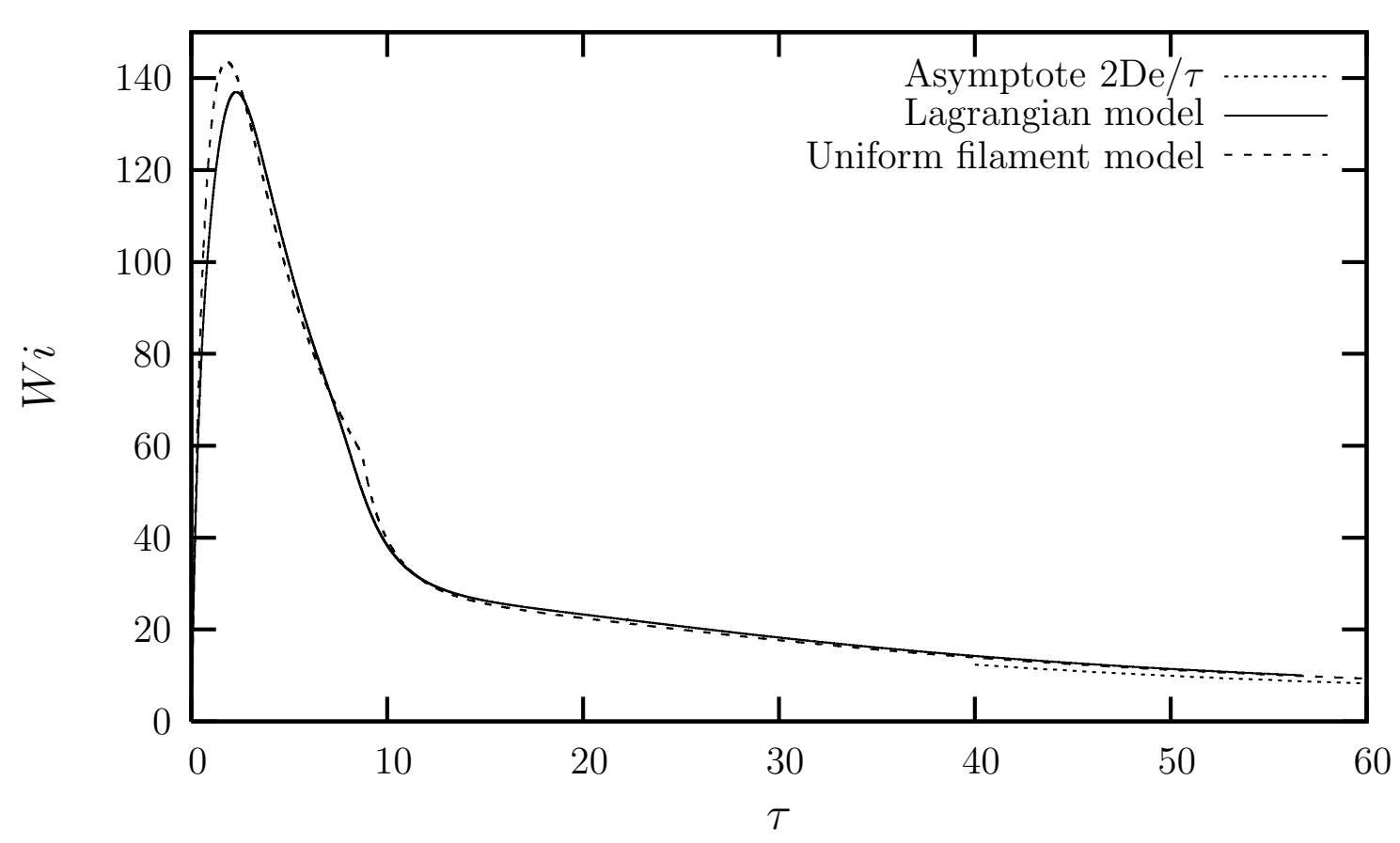

Figure 21: 